\title{
CRUISE: Cortical reconstruction using implicit surface evolution
}

\author{
Xiao Han, ${ }^{\mathrm{a}}$ Dzung L. Pham, ${ }^{\mathrm{b}}$ Duygu Tosun, ${ }^{\mathrm{a}}$ Maryam E. Rettmann, ${ }^{\mathrm{c}}$ \\ Chenyang $\mathrm{Xu}^{\mathrm{d}}{ }^{\mathrm{d}}$ and Jerry L. Prince, ${ }^{\mathrm{a}, *}$ \\ ${ }^{a}$ Department of Electrical and Computer Engineering, Johns Hopkins University, Baltimore, MD 21218 United States \\ ${ }^{\mathrm{b}}$ Department of Radiology, Johns Hopkins University, Baltimore, MD 21287, United States \\ ${ }^{c}$ Laboratory of Personality and Cognition, National Institute on Aging, Baltimore, MD 21224, United States \\ ${ }^{\mathrm{d}}$ Imaging and Visualization Department, Siemens Corporate Research, Princeton, NJ 08540, United States
}

Received 23 January 2004; revised 19 May 2004; accepted 24 June 2004

Available online 8 October 2004

\begin{abstract}
Segmentation and representation of the human cerebral cortex from magnetic resonance (MR) images play an important role in neuroscience and medicine. A successful segmentation method must be robust to various imaging artifacts and produce anatomically meaningful and consistent cortical representations. A method for the automatic reconstruction of the inner, central, and outer surfaces of the cerebral cortex from T1-weighted MR brain images is presented. The method combines a fuzzy tissue classification method, an efficient topology correction algorithm, and a topology-preserving geometric deformable surface model (TGDM). The algorithm is fast and numerically stable, and yields accurate brain surface reconstructions that are guaranteed to be topologically correct and free from selfintersections. Validation results on real MR data are presented to demonstrate the performance of the method.

(c) 2004 Elsevier Inc. All rights reserved.
\end{abstract}

Keywords: Magnetic resonance; T1-weighted MR brain images; Cerebral cortex; Cortical reconstruction; Human brain mapping

\section{Introduction}

Accurate reconstruction of the cerebral cortex from magnetic resonance (MR) images plays an important role in neuroscience. Reconstructed cortices enable the visualization and study of the sulcal and gyral patterns of an individual subject (Carman et al., 1995; Essen et al., 2001; Fischl et al., 1999a,b; Thompson et al., 2001) and allow morphometric measurements such as volume (Kim et al., 2000), surface area (Magnotta et al., 1999), thickness (Kruggel et al., 2003; Yezzi and Prince, 2003), and sulcal depth (Manceaux-Demiau et al., 1998). These measures provide valuable

\footnotetext{
* Corresponding author. Department of Electrical and Computer Engineering, The Johns Hopkins University, 3400 North Charles Street, Baltimore, MD 21218. Fax: +1 4105165566.

E-mail address: prince@jhu.edu (J.L. Prince).

Available online on ScienceDirect (www.sciencedirect.com.)
}

information about the cortical characteristics of both normal development and pathological disease. Correspondences between cortical reconstructions from different subjects can also be used for image registration (Cachier et al., 2001; Davatzikos, 1997; Hellier and Barillot, 2003; Tao et al., 2002), digital atlas labeling (Jaume et al., 2002; Sandor and Leahy, 1997), and population-based probabilistic atlas generation (Thompson et al., 1997). In addition, cortical reconstruction is important for functional brain mapping (Dale and Sereno, 1993), surgical planning (Grimson et al., 1998), and cortical unfolding or flattening (Carman et al., 1995; Drury et al., 1996; Essen et al., 2001; Fischl et al., 1999a,b; Tosun and Prince, 2001).

Geometrically, the cerebral cortex is a thin, folded sheet of gray matter (GM) that is $1-5 \mathrm{~mm}$ thick, with an average thickness of approximately $2.5 \mathrm{~mm}$ (Beatty, 2001; Griffin, 1994; von Economo, 1929; Zilles, 1990). As illustrated in Fig. 1, the cortical GM is bounded by the cerebrospinal fluid (CSF) on the outside, forming the outer (or pial) cortical surface, and by the white matter (WM) on the inside, forming the inner cortical surface. It is useful to define the central cortical surface as well; it lies at the geometric center between the inner and outer surfaces, approximating cytoarchitectonic layer 4 and representing an overall two-dimensional (2-D) approximation to the three-dimensional (3-D) cortical sheet.

We define cortical reconstruction to be the localization and representation of one or more of these cortical surfaces. This is a step beyond segmentation, which only classifies the image pixels as belonging to cortical GM or not. An accurate cortical reconstruction must generate a geometric representation of the cortex that is consistent with the true geometry of the brain cortex, complete with multiple lobes, gyral folds, and narrow sulci. This task is made difficult due to artifacts such as image noise, partial volume effects, and intensity inhomogeneities. Image noise, for example, often produces segmentation artifacts that incorrectly classify cortical GM, resulting in extra or missing components or representations that are flawed from an anatomical standpoint (e.g., having holes or handles). MR image intensity inhomogeneities, caused by nonuniform radiofrequency 


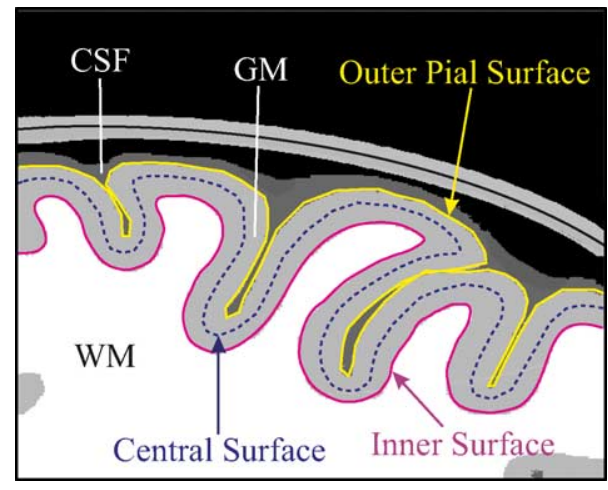

Fig. 1. A cartoon drawing illustrating the definition of the three cortical surfaces.

coil sensitivity patterns, can lead to inaccurate thickness patterns or even missing pieces of cortex if not addressed carefully. Partial volume averaging is particularly problematic in "tight" sulci, where cortical GM is virtually "back-to-back," having little CSF between the two GM banks. Poorly reconstructed cortex (usually the pial surface, but sometimes the central surface as well) will be "bridged" across the sulci causing a flawed representation and erroneously derived geometric measures such as thickness.

Topology is an important consideration in generating cortical reconstructions. A surface corresponding to a single cortical hemisphere should be topologically equivalent to a disc. It is customary in cortical reconstruction to connect the two hemispheres with a "tube" passing around the corpus callosum and through the brain stem at the level of the diencephalon. In this case, a cortical surface should be topologically equivalent to a sphere. It should also be a regular surface, that is, smooth and devoid of self-intersections. These surface properties-topologically equivalent to a sphere, smooth, and having no selfintersections - are difficult to maintain simultaneously in practice. Reconstructing a smooth surface is usually accomplished by using a deformable surface model having internal "tension" or "bending" forces (Dale et al., 1999; Goldenberg et al., 2002; MacDonald et al., 2000; Xu et al., 1999; Zeng et al., 1999). We follow this approach here as well. Generating a topologically correct cortical surface can be accomplished by first finding an initial surface with the correct topology and then preserving the topology during surface evolution using a parametric deformable model (Dale et al., 1999; MacDonald et al., 2000; Xu et al., 1999). The deforming surface in parametric deformable models, however, can easily develop self-intersections; the detection and prevention of which is usually very time-consuming (Dale et al., 1999; MacDonald et al., 2000). Methods that use geometric or level-set deformable models (Zeng et al., 1999; Goldenberg et al., 2002) automatically avoid the self-intersection problem, but the topology of the final result can be arbitrary. The present work differs from previous approaches in that we employ a new topology-preserving geometric deformable model (TGDM; Han et al., 2003), which maintains topology like parametric deformable models but retains the advantage of geometric deformable models of automatically generating simple surfaces (i.e., no selfintersections) without special computational constraints.

In this paper, we present our method for reconstructing the inner, central, and pial cortical surfaces from MR images of human brain. The proposed 3-D reconstruction method, called
Cortical Reconstruction using Implicit Surface Evolution (CRUISE), cascades a fuzzy segmentation method, a topology correction algorithm, and a topology-preserving geometric deformable surface model to reconstruct these three surfaces. CRUISE is accurate, robust, anatomically consistent, and computationally efficient and guarantees the proper nesting of all three surfaces. The first step in CRUISE involves a brief, manual preprocessing procedure; thereafter, CRUISE is fully automatic. In Methods, we detail the various steps of CRUISE. In Results, we describe the results of several qualitative and quantitative validation experiments based on visual inspection, manually placed landmarks, and reproducibility. In Discussion, we compare CRUISE to other published cortical reconstruction methods. Finally in Conclusion and future work, we summarize the contributions of this work and propose some directions for future work.

We note that preliminary versions of this work have appeared in two conference papers (Han et al., 2001c; Pham et al., 2002).

\section{Methods}

A block diagram of CRUISE is shown in Fig. 2. We now describe each step in detail.

\section{Preprocessing}

CRUISE starts with T1-weighted (spoiled gradient echo) volumetric MR brain images with voxel size $0.9375 \times 0.9375 \times$ $1.5 \mathrm{~mm}$. As the first step of the process (see Fig. 2), an input

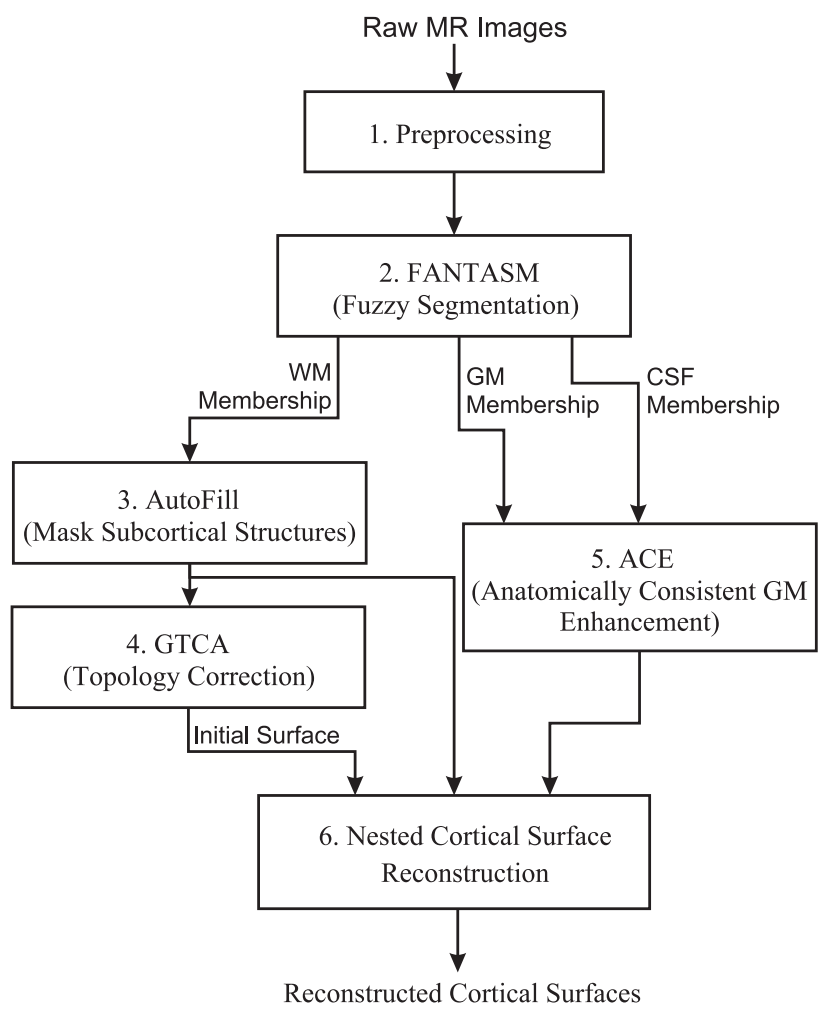

Fig. 2. Block diagram of the overall cortical surface reconstruction system. 
brain image is preprocessed to remove skin, bone, fat, and other noncerebral tissues using a semiautomated software package developed by Christos Davatzikos and Jerry Miller at the Johns Hopkins University (Goldszal et al., 1998). Fig. 3 shows two slices from a typical MR image volume before and after this procedure was applied. The increased contrast apparent in the last two images is simply the result of an image intensity rescaling.

After "skull-stripping," the cerebellum and brain stem are removed manually, and the anterior commissure (AC), posterior commissure (PC), and midsagittal vertical plane are manually identified. The remaining volume is then resliced in "Talairach" orientations (Talairach and Tournoux, 1988) using cubic-spline interpolation, yielding cubic voxels having the in-plane resolution in all three directions $(0.9375 \times 0.9375 \times 0.9375$ $\mathrm{mm})$. This reduces the directional dependency in subsequent processing. Upsampling the data further might help achieve more accurate surface reconstruction results but would also significantly increase the overall computational cost.

Some of the preprocessing steps require manual intervention in our current implementation. We note that further automation in these steps should be possible using published methods (Kapur et al., 1996; Kruggel and von Cramon, 1999; Shattuck and Leahy, 2002). The preprocessing step takes between 5 and $10 \mathrm{~min}$, depending on the skill and experience of the operator.

\section{Tissue classification}

After preprocessing, the second step of CRUISE (see Fig. 2) applies a tissue classification algorithm to identify the spatial distribution of GM, WM, and CSF within the image volume. Like several other published methods, we use a "fuzzy" segmentation approach, which yields three membership images that represent the proportion of each tissue type within each voxel. Our approach, called Fuzzy And Noise Tolerant Adaptive Segmentation Method (FANTASM), extends our previous method, adaptive fuzzy Cmeans (AFCM) (Pham and Prince, 1999), by incorporating a spatial smoothing term to reduce the effects of noise. It also compensates for MR intensity inhomogeneities by estimating a gain field along with the segmentation, as in AFCM. We briefly describe FANTASM here.

Let $\Omega$ be the set of voxel indices, $C$ be the number of tissue classes, and $y_{j}, j \in \Omega$ be the observed (preprocessed) MR image values. The goal of FANTASM is to find intensity centroids $v_{k}, k=1, \ldots, C$, a gain field $g_{j}, j \in \Omega$, and membership values $u_{j k}, j \in \Omega, k=1, \ldots, C$ that will minimize the following objective function (Pham, 2001)

$J_{\mathrm{FANTASM}}=J_{\mathrm{AFCM}}+\frac{\beta}{2} \sum_{j \in \Omega} \sum_{k=1}^{C} u_{j k}^{q} \sum_{l \in N_{j}} \sum_{m \neq k} u_{l m}^{q}$

where (Pham and Prince, 1999):

$$
\begin{aligned}
J_{\mathrm{AFCM}}= & \sum_{j \in \Omega} \sum_{k=1}^{C} u_{j k}^{q}\left\|y_{j}-g_{j} v_{k}\right\|^{2}+\lambda_{1} \sum_{j \in \Omega} \sum_{r=1}^{3}\left(D_{r} * g\right)_{j}^{2} \\
& +\lambda_{2} \sum_{j \in \Omega} \sum_{r=1}^{3} \sum_{s=1}^{3}\left(D_{r} * D_{s} * g\right)_{j}^{2}
\end{aligned}
$$

There are several terms in Eqs. (1) and (2) that require explanation. The parameter $q$, which must satisfy $q>1$ (and is set to 2 in this work), determines the amount of "fuzziness" of the resulting classification (Bezdek et al., 1993). The number of classes $C$ is 3 , corresponding to GM, WM, and CSF. The parameters $\beta, \lambda_{1}$, and $\lambda_{2}$ are weights that determine the amount of smoothness in resulting membership functions $(\beta)$ and the gain field $\left(\lambda_{1}\right.$ and $\left.\lambda_{2}\right)$. These parameters are set empirically, and for the results reported in this paper, we used $\beta=150, \lambda_{1}=2 \times 10^{4}$, and $\lambda_{2}=2 \times 10^{5}$. The symbol $N_{j}$ represents the set of first order neighbors of pixel $j$. The expressions $D_{r} * g$ and $D_{r} * D_{s} * g$ represent first- and second-order finite differences applied to the gain field; details on this notation can be found in Pham and Prince (1999)

The FANTASM objective function in Eq. (1) adds a novel "fuzzy" spatial smoothing term to the AFCM objective function, providing better regional continuity of tissue classification (see also Pham, 2001). Although this smoothing term resembles the prior probability models used in Markov random fields (Zhang, 1992), the particular form we use here yields an appealing EulerLagrange equation that simplifies minimization of the objective function, which is achieved using an iterative algorithm similar to that in Pham and Prince (1999).

Fig. 4 shows the results of applying standard fuzzy $C$-means (FCM), AFCM, and FANTASM to a simulated MR image (Collins et al., 1998). The FCM result shows sensitivity to both image intensity inhomogeneity and noise artifacts, while the AFCM result is better because it adapts to intensity inhomogeneities. FANTASM remains robust to intensity inhomogeneities and adds a visually apparent spatial smoothness to the membership values. We have shown in simulation experiments that FANTASM also produces more accurate segmentations than AFCM or FCM and is

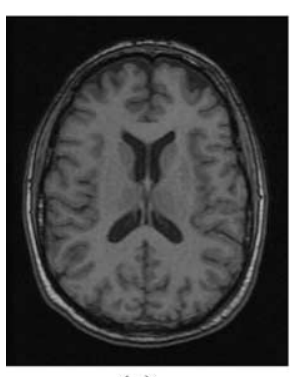

(a)

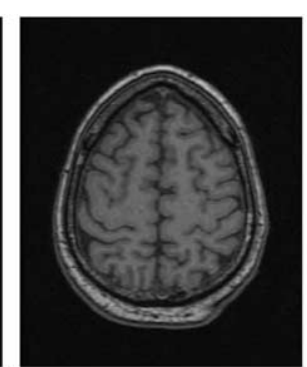

(b)

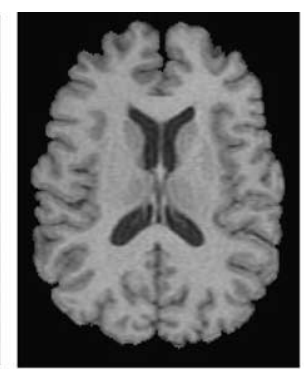

(c)

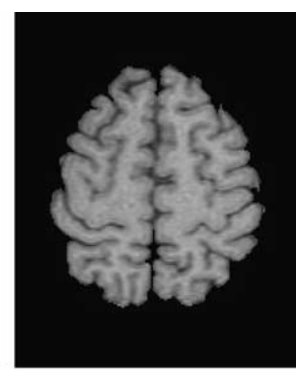

(d)

Fig. 3. Sample slices from acquired MR image data and preprocessed data: (a, b) two slices from original MR acquisition, (c, d) same slices after preprocessing to extract cerebrum. 


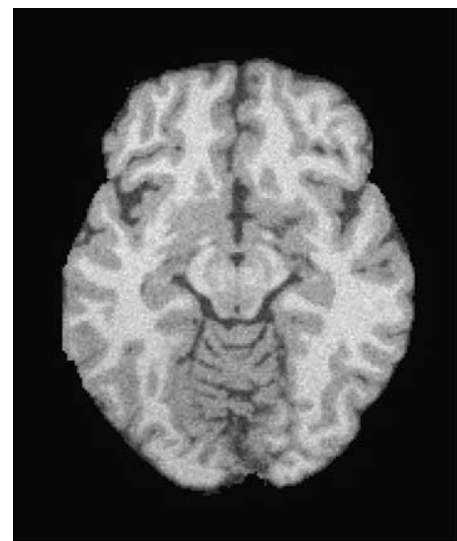

(a)

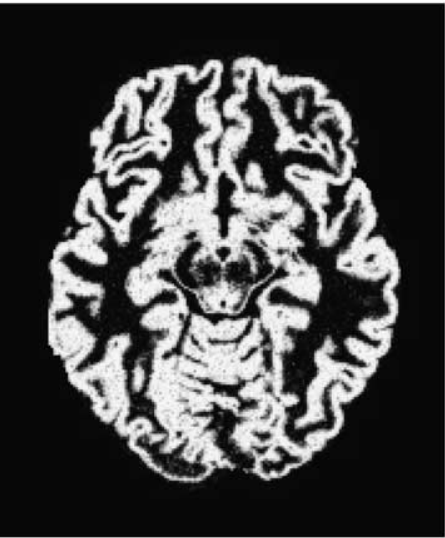

(d)

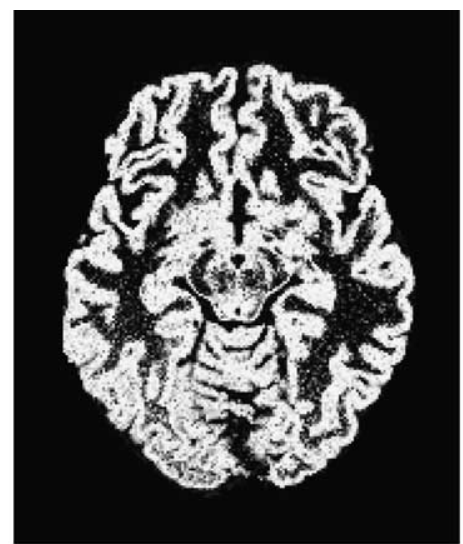

(b)

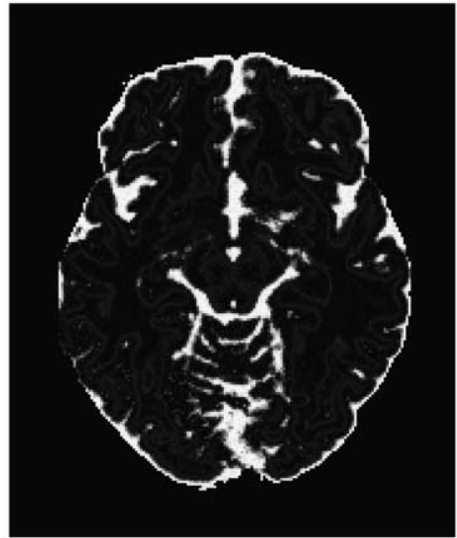

(e)

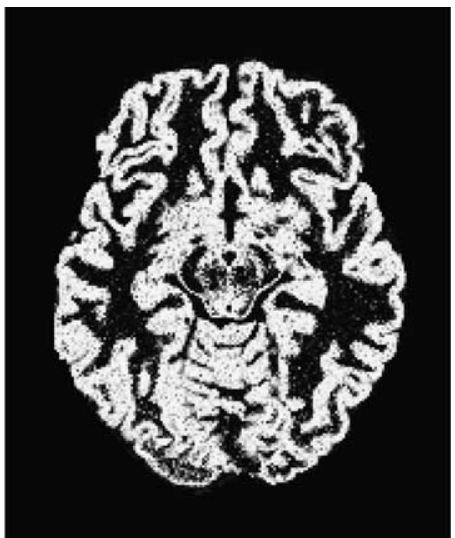

(c)

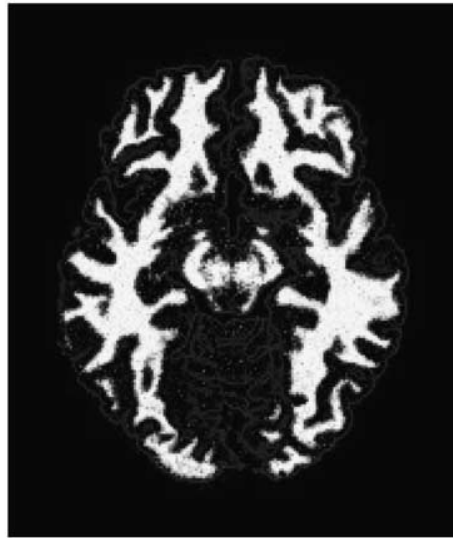

(f)

Fig. 4. Tissue classification: (a) MR image corrupted by noise and intensity inhomogeneities, (b) FCM GM membership, (c) AFCM GM membership, (d) FANTASM GM membership, (e) FANTASM CSF membership, (f) FANTASM WM membership.

comparable (or in some cases better) than other leading soft segmentation approaches in the literature.

\section{Automatic editing of the WM membership function}

From Fig. 4f, we see that the "outside" of the WM membership function is a good approximation to the inner cortical surface. In fact, our approach in the next section starts with an isosurface algorithm that finds this approximation. However, it is also apparent from Fig. 4f that finding the "outside" of the WM membership function will also find subcortical interfaces near the brainstem and even within the ventricles, which are not "cortical." In this section, we describe AutoFill (Step 3 in Fig. 2), an automatic method that edits the WM membership function, filling the ventricles and subcortical GM structures (e.g., putamen, caudate nucleus, thalamus, hypothalamus) with WM membership values (see also Han et al., 2001b). In this way, the outside of the edited WM membership approximates the inner cortical surface, connecting the two hemispheres around the corpus callosum (superior connection) and through the brain stem at the level of the diencephalon (inferior connection).

AutoFill uses the fact that the subcortical GM together with the ventricles form concavities inside the WM that open from the bottom of the brain. We find the ventricles using a standard 3-D geometric deformable model (GDM) (Sethian, 1999; Xu et al., 2001), initialized using a small sphere positioned at the centroid of the
CSF membership volume. The GDM uses forces including both the conventional curvature force, which provides a small amount of surface smoothing, and a regional signed pressure force (Xu et al., 2001) that drives the surface toward the boundary of the ventricles. The signed pressure force is derived from the CSF membership function, causing the surface to expand at places where the membership value is larger than 0.5 and contract if less than 0.5 . The curvature force also prevents the surface from growing into the CSF outside the GM through some thin connections at the bottom of the brain. A typical result is shown in Fig. 5a.

The ventricles are then used as "seeds" to fill the WM membership function using 2-D region growing in successive coronal slices, as shown in Fig. 5b. Sealing lines, roughly corresponding to the bottom of the diencephalon, are automatically identified at the base of the brain within each coronal slice to constrain the region-growing algorithm. Region growing may fail when a thin strand of WM that exists between the putamen and the insular GM is broken due to an inaccuracy in the tissue classification. We address this by applying a mask to identify the putamen region and constraining the region growing to take place within this mask, as shown in Fig. 5c. The mask is fixed in Talairach coordinates (Talairach and Tournoux, 1988) and is mapped automatically to each individual brain after the brain is registered to the standardized space (Lemoine et al., 1991). Fig. 5d shows an isosurface of an AutoFill-edited WM membership function. 


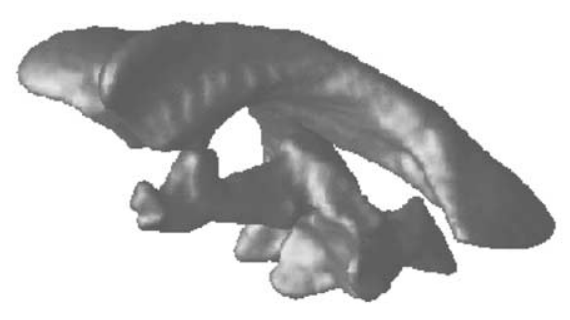

(a)

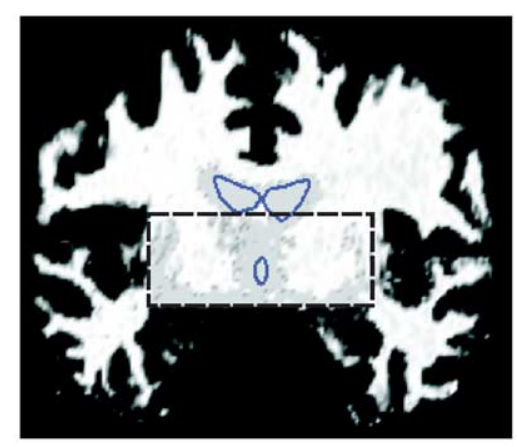

(c)

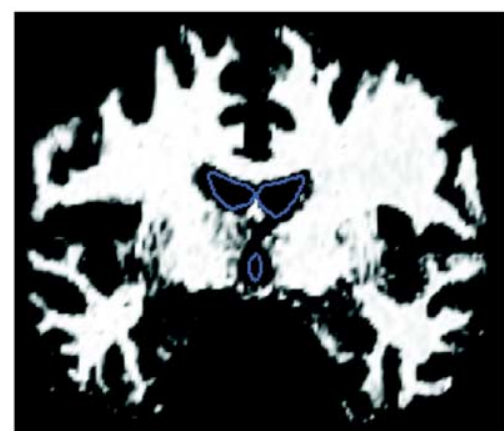

(b)

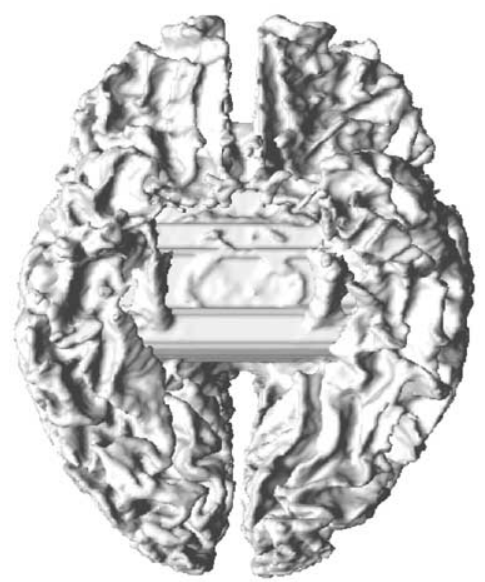

(d)

Fig. 5. AutoFill: (a) deformable model segmentation of ventricles, (b) a coronal slice showing the region-growing "seed," (c) putamen mask and regiongrowing result, (d) isosurface of the edited WM membership function.

\section{Topology correction of WM isosurface}

An isosurface of the edited WM membership function (we use an isovalue of 0.5 ) yields a close approximation to the inner cortical surface. Because of noise and partial volume averaging, however, this surface does not, in general, have the correct spherical topology. In fact, typically, there are hundreds of handles (a handle is like that of a coffee cup), which is incompatible with cortical anatomy. Topology correction has proven to be a challenging task in the past (Dale et al., 1999; Shattuck and Leahy, 1999; Xu et al., 1999). A few practical algorithms (Fischl et al., 2001; Kriegeskorte and Goebel, 2001; Shattuck and Leahy, 2001), including our own graph-based topology correction algorithm (GTCA) (Han et al., 2001a, 2002), have recently emerged to solve this problem. Here, we briefly describe GTCA, which was developed for binary-valued volumes, and we show here how it is readily extended to correct topology of isosurfaces of the edited WM membership function, which is a continuous-valued volume. The WM isosurface topology correction constitutes Step 4 of CRUISE (see Fig. 2).

GTCA uses digital topology principles to detect and remove handles, which implies that it works on binary-valued volumes. It further assumes that the binary volume consists of a single binary object without any internal cavities. ${ }^{1}$ To adhere to these assumptions, a WM membership function volume must be first thresholded (at membership value 0.5 ) to create a binary volume

\footnotetext{
${ }^{1}$ Unlike handles, cavities refer to the hollow parts within an otherwise solid object that are fully surrounded by the object.
}

consisting of object (or foreground) and background. The object is then processed to keep only the largest connected component, and cavities, if they exist, are filled by region growing.

GTCA is then applied on the resulting (binary) object to remove all its handles (Han et al., 2001a, 2002). It alternatively applies two types of topology correction filters: a foreground filter cuts the handles and a background filter fills the tunnels (also called holes) associated with the handles. The filters are first applied at a fine scale to cut off thin handles and fill up narrow tunnels, and the scale is increased if necessary until all the handles/tunnels are removed. The topology correction filters are designed so that the cut (or fill) is always located at a thin part of each handle (or tunnel), in an attempt to minimize the changes that are made to the binary object. GTCA can efficiently remove all the handles from a WM segmentation with hundreds or even thousands of handles, and the average number of voxels changed per handle is typically less than three (Han et al., 2001a, 2002). This small average number agrees with the fact that handles are typically caused by imaging noise and/or partial volume effects and thus are usually small in size, as also shown in Fig. 6a.

As described above, GTCA will correct the underlying binary volume implied by the 0.5 -isosurface of the edited WM membership function. But by "binarizing" the membership function, we have apparently lost the subvoxel accuracy when computing the isosurface of the WM membership function. Fortunately, it is relatively straightforward to extend GTCA in order to address this problem and recover the subvoxel accuracy in computing the final topology-corrected isosurface. Such an extension is summarized in 


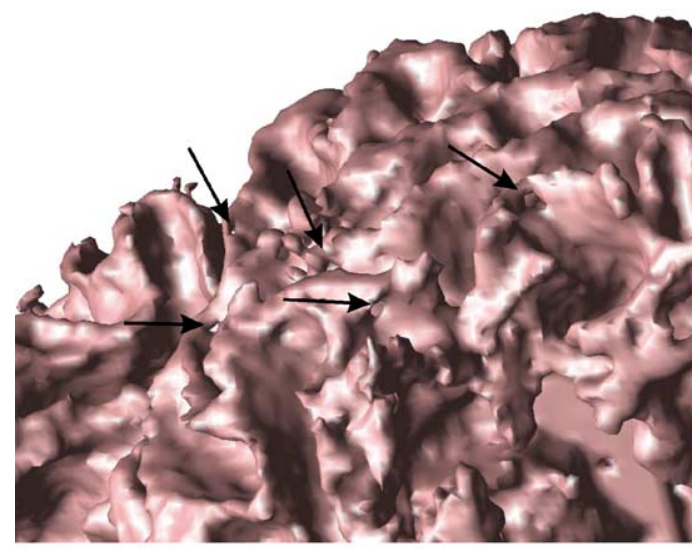

(a)

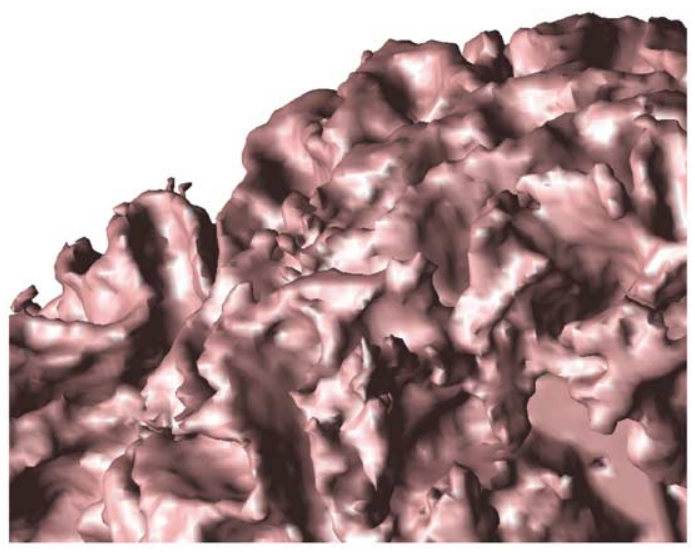

(b)

Fig. 6. WM boundary surface obtained (a) before and (b) after topology correction. The black arrows in (a) indicate handle locations.

the following algorithm, which is used to get a topologically correct surface representing the inner surface of the cortex (connected across the hemispheres). The surface maintains the subvoxel accuracy of the initial 0.5 -isosurface of the edited WM membership function except at topology corrections.

Algorithm 1. (WM isosurface topology correction).

1. Binarize $\mu_{\mathrm{WM}}^{\prime}$, the edited $\mathrm{WM}$ membership function after AutoFill, at the threshold 0.5 to get a binary volume $I_{\text {binary }}$.

2. Clean $I_{\text {binary }}$ to keep only the largest connected foreground component and then fill the cavities if applicable. The output is denoted as $I_{\mathrm{tmp}}$.

3. Run GTCA on the volume $I_{\mathrm{tmp}}$ to get the topology corrected volume $I_{\text {correct }}$.

4. Compare the binary volumes $I_{\text {binary }}$ and $I_{\text {correct. If a foreground }}$ point in $I_{\text {binary }}$ is changed to background in $I_{\text {correct, }}$ then we reduce the membership value at this point in $\mu_{\mathrm{WM}}^{\prime}$ to a value below 0.5 , for example, we can simply set it to 0 . If a background point in $I_{\text {binary }}$ is changed to foreground in $I_{\text {correct, }}$, then we modify the membership volume $\mu_{\mathrm{WM}}^{\prime}$ at this point to a value above 0.5 , for example, set it to 1 . The modified volume is denoted by $\mu_{\mathrm{WM}}^{\prime \prime}$.

5. The 0.5-isosurface of the new continuous-valued volume $\mu_{\mathrm{WM}}^{\prime \prime}$ gives the topologically corrected WM boundary surface.

We note that in the last step of the algorithm, we use the Connectivity Consistent Marching Cubes (CCMC) algorithm described in Han et al. (2003) to compute the isosurface, which guarantees that the extracted isosurface has a topology consistent with the digital topology principles used in GTCA.

As a visual demonstration of the topology correction result, in Fig. 6, we show magnified views of a WM isosurface before and after the topology correction. All handles seen in panel (a) are removed in panel (b), and the surface in (b) is exactly the same as that in (a) except where the handles have been removed.

\section{Anatomically consistent GM enhancement}

As described above, the output of Step 4 in Fig. 2 is a topologically correct representation of the GM/WM surface. This serves as an initial surface for finding the inner, central, and outer layers of the cortex in Step 6, which are found using a deformable surface model method (see Nested cortical surface reconstruction). However, we (and some other groups) have found that partial volume averaging at voxels of cortical GM, particularly in the sulcal regions where the cortex is "back-to-back," is responsible for wildly inaccurate estimates of both the central and outer surfaces, unless directly addressed in some fashion. Some groups have used cortical thickness constraints to produce a reasonable result (MacDonald et al., 2000; Zeng et al., 1999). We have taken another approach, called Anatomically Consistent Enhancement (ACE), represented by Step 5 in the block diagram. We note that the approach described here improves significantly upon our preliminary ACE approaches, which were reported in $\mathrm{Xu}$ et al. (2000) and Han et al. (2001c).

The goal of ACE is to provide a GM representation that has evidence of sulci where it might not otherwise exist due to the partial volume effect. Accordingly, ACE modifies the initial GM segmentation to create a thin, digital separation between sulcal GM banks. The basic approach, as illustrated in Fig. 7, is to automatically locate the exterior skeleton of the GM/WM interface and reduce the GM membership value on the skeleton, thus changing this particular GM into CSF. It is clear from Fig. 7a, however, that use of the conventional Euclidean skeleton will "gouge" the GM indiscriminantly, regardless of the presence of actual CSF. This can be improved by incorporating the presence of CSF in the definition of distance used to compute the outer

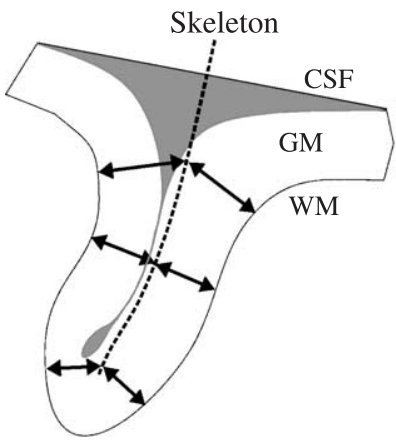

(a)

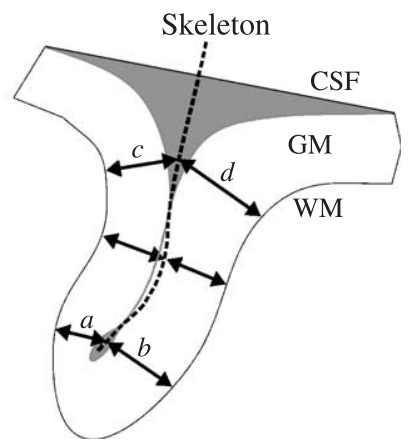

(b)
Fig. 7. (a) The true skeleton under Euclidean distance measure. (b) The preferred skeleton location for ACE. 
skeleton, as illustrated in Fig. 7b where a weighted distance measure will essentially imply that $a=b$ and $c=d$ (a similar approach can be found in Rivière et al., 2002). In sulci where there is no evidence of CSF, the distance will default back to the Euclidean definition, and the gyral banks will be split equally-a reasonable assumption given no other knowledge.

The weighted distance function $D(x)$, where $x$ is a 3-D spatial position, can be computed by solving the following Eikonal equation:

$F(\mathbf{x})\|\nabla D(\mathbf{x})\|=1$ in $\Omega$,

$D(\mathbf{x})=0$ for $\mathbf{x} \in \Gamma$,

where $F(\mathbf{x})$ is a spatially varying speed function defined within the computational domain $\Omega$, and $\Gamma$ is the GM/WM interface to which the distance is measured. This equation can be solved using the fast marching method (FMM) (Sethian, 1999), an efficient algorithm that marches the initial boundary as a moving front according to the speeds prescribed by $F(\mathbf{x})$. If the speed function $F(\mathbf{x})$ is unity at every point, then $D(\mathbf{x})$ is the usual Euclidean distance.

We would like to make the distances computed in the CSF to be much larger than the usual Euclidean distance. Accordingly, we define the speed function as

$F(\mathbf{x})=1-0.9 \mu_{\mathrm{csf}}(\mathbf{x})$,

where $\mu_{\text {csf }}(\mathbf{x})$ is the CSF fuzzy membership function. Using this definition, the moving front will slow down in CSF, and the outside skeleton of the GM/WM interface will be shifted as depicted in Fig. 7b. For $\Gamma$, the GM/WM interface, we use the 0.5 isosurface of the WM membership function but smooth it slightly using a geometric curvature flow (Sethian, 1999) to avoid extraneous branches in the computed skeleton.

The GM membership function $\mu_{\mathrm{GM}}$ is modified only at the skeletal points exterior to the GM/WM interface, which can be easily located. We first note that mathematically, Eq. (3) tells us that $F(\mathbf{x})|| \nabla D(\mathbf{x}) \|=1$ throughout the computational domain. The skeletal points, however, form so-called "shocks" of this equation, at which the gradient $\nabla D(\mathbf{x})$ is not well defined. Finite difference approximations to $\nabla D(\mathbf{x})$ can still be computed, of course, and their values can actually distinguish the shocks from other points.
For example, a centered finite difference approximation to $F(\mathbf{x}) \| \nabla D(\mathbf{x})||$ will yield values much smaller than 1 on the shocks. Thus, after $D(\mathbf{x})$ is computed using the FMM method, we reevaluate $\nabla D(\mathbf{x})$ using the centered finite difference operator and define the exterior skeleton as the set of points: $S=$ $\{x|F(\mathbf{x})| \mid \nabla D(\mathbf{x}) \| \leq T$ and $\mathbf{x}$ is outside the GM/WM interface $\}$. $T$ is a threshold less than 1 , which we empirically set to 0.8 . We further perform a morphological thinning operation on the initial set $S$ to make sure that the skeleton is no more than one voxel thick. We then define the modified GM membership function by

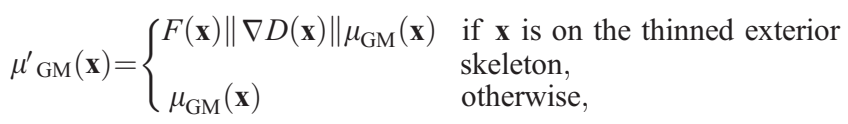

where $\nabla D(\mathbf{x})$ is understood to represent a finite difference approximation to the gradient. Note that, although the modification of GM takes place at the voxel level, the cortical surface estimate has subvoxel accuracy and can stay back-to-back within a tight sulcus, as demonstrated in Fig. 10 in the next section.

A cross-sectional view of a GM membership function before and after ACE modification is shown in Fig. 8. We observe that there are many examples of GM modifications taking place in the sulci within this cross-section. In most cases where sulcal banks are back-to-back and no CSF was apparent in the original GM membership image, there is now a noticeable gap between the banks in the modified membership image. There are also several examples showing a "gentle reinforcement" of weak sulci, preserving the location of the sulcal gap and the nonuniform cortical thickness on opposite banks.

\section{Nested cortical surface reconstruction}

The previous steps have set the stage for the estimation of three cortical surfaces using a special deformable surface model method, which we refer to as nested cortical surface reconstruction and is the final step of CRUISE (Step 6 in Fig. 2). We begin by describing TGDM, a topology-preserving geometric deformable surface model, which is at the core of this process (Han et al., 2003).

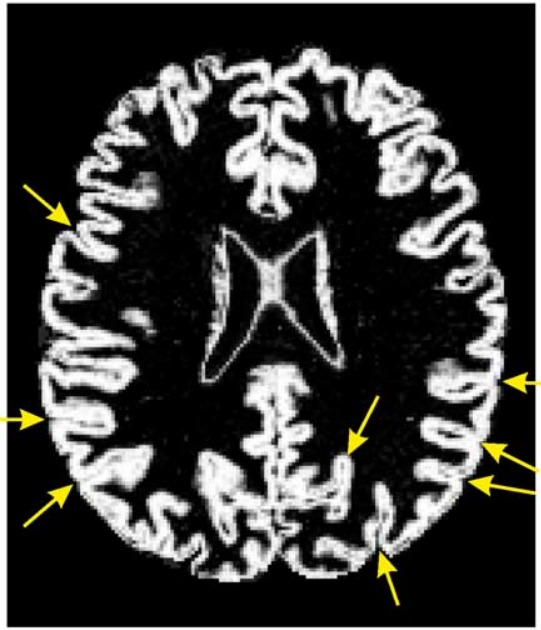

(a)

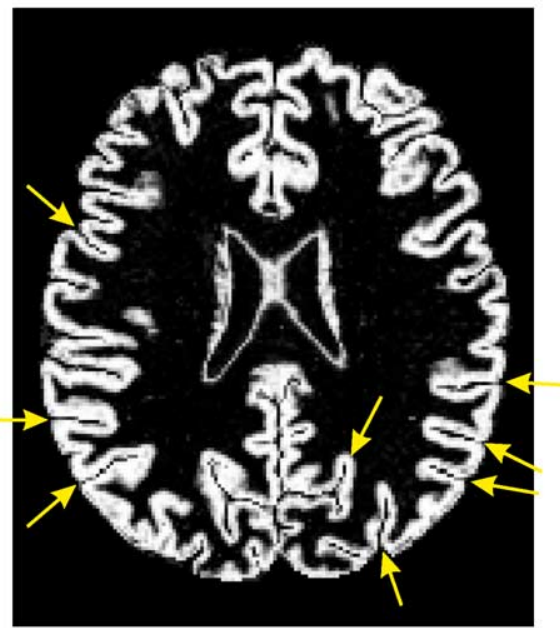

(b)

Fig. 8. (a) Original GM membership function and (b) the ACE enhanced GM membership function. 
We then describe the specifics involved in the estimation of each of the three surfaces, including the process that assures the surfaces properly nest among each other.

\section{Topology-preserving geometric deformable surface model (TGDM)}

In geometric deformable surface models, the evolving surface is embedded as the zero level set of a higher dimensional level set function $\Phi(\mathbf{x}, t): U \times R^{+} \rightarrow R$, where $U \subset R^{3}$, and propagates implicitly through the temporal evolution of $\Phi$. By convention, $\Phi$ is a signed distance function to the surface with negative value inside the surface and positive outside, which can be computed by first solving Eq. (3) with a constant speed function and then assigning negative signs to values inside the surface. In a standard geometric deformable model, the evolution of the level set function $\Phi$ is usually prescribed by a partial differential equation of the following form (cf. Sethian, 1999):

$$
\begin{aligned}
\Phi_{t}(\mathbf{x}, t)= & F_{\text {prop }}(\mathbf{x})\|\nabla \Phi(\mathbf{x}, t)\|+F_{\text {curv }}(x)\|\nabla \Phi(x, t)\| \\
& +\vec{F}_{\text {adv }}(x) \cdot \nabla \Phi(x, t),
\end{aligned}
$$

where $\Phi_{t}$ denotes the partial derivative of $\Phi$ with respect to its time variable, and $\nabla \Phi$ is the spatial gradient of $\Phi . F_{\text {prop }}, F_{\text {curv }}$, and $\vec{F}_{\text {adv }}$ are speed terms that can be spatially varying. In general, $F_{\text {prop }}$ is an expansion or contraction speed, $F_{\text {curv }}$ depends on intrinsic geometry of the surface, and $\vec{F}_{\text {adv }}$ is a velocity field that tries to move the contour in a certain direction. In this work, $F_{\text {prop }}$ is always taken to be a signed pressure force $R(\mathbf{x})$ computed from the fuzzy membership functions, $F_{\text {curv }}$ is set to be proportional to the mean curvature $\kappa(\mathbf{x})$ of the surface, and $\vec{F}_{\text {adv }}$, when applied, is taken to be a gradient vector flow (GVF) field $\vec{v}(\mathbf{x})$ (Xu and Prince, 1998). With these choices, the evolution equation becomes

$$
\begin{aligned}
\Phi_{t}(\mathbf{x}, t)= & \omega_{R} R(\mathbf{x})\|\nabla \Phi(\mathbf{x}, t)\|+\omega_{\kappa} \kappa(\mathbf{x})\|\nabla \Phi(\mathbf{x}, t)\| \\
& +\omega_{\vec{v}} \vec{v}(x) \cdot \nabla \Phi(x, t),
\end{aligned}
$$

where $\omega_{R}, \omega_{\kappa}$, and $\omega_{\vec{v}}$ are the weights that cause the respective speeds to be emphasized differently. Different specific choices of the signed pressure force, the GVF force, and the weights produce deformable models that aim at different boundaries of the brain cortex, as described later.

The numerical solution of Eq. (7) can be obtained by approximating the time derivative by a forward difference and the spatial derivatives on the right-hand side by upwind numerical schemes (for details, see Sethian, 1999). This yields

$\Phi\left(\mathbf{x}, t_{m+1}\right)=\Phi\left(\mathbf{x}, t_{m}\right)+\Delta t \Delta \Phi\left(\mathbf{x}, t_{m}\right)$,

where $\mathbf{x}$ now denotes a voxel location, $\Delta \Phi$ represents the upwind numerical approximation to the right-hand side of Eq. (6), $t_{m}$ and $t_{m+1}$ denote two consecutive time steps, and $\Delta t=t_{m+1}-t_{m}$ is the time step-size. Starting from an initial level set function $\Phi(\mathbf{x}, 0)$ that embeds the initial surface, Eq. (8) is iteratively solved until convergence or until stopped after a specified number of time steps. For efficiency, we adopt the narrow band approach, which updates the level set function only at a small set of points in the neighborhood of the zero level set at each time step instead of at all the points in the computational domain (Sethian, 1999).

In nested cortical surface reconstruction, the surfaces cannot be allowed to change topology during their deformation. Since traditional GDMs readily change topology, they are not suitable in this application unless modified. Recently, we introduced the topologypreserving geometric deformable model (TGDM), which introduces a constraint during the numerical implementation of a GDM that prevents the evolving implicit surface from ever changing topology (Han et al., 2003). The constraint is implemented by checking the topology of a digital object implied by the evolving level set function and restricting the level set function from changing sign if the sign change will cause the digital object to change topology. Making this check is compatible with the standard narrow band implementation and adds only about $7 \%$ computational overhead. The resulting surface still has subvoxel resolution and is guaranteed to be a simple surface without any self-intersections.

\section{Inner cortical surface}

Step 4 of CRUISE (see Fig. 2) yields a topologically correct estimate of the GM/WM interface. Because of both noise and the "abrupt" changes that are made to correct topology, this surface is typically a bit rougher than desired. Thus, we apply TGDM with a curvature force and a signed pressure force given by

$R_{\text {in }}(\mathbf{x})=2 \mu_{\mathrm{WM}}^{\prime}(\mathbf{x})-1$

to find a slightly smoother surface that stays close to the 0.5 isosurface of the WM membership function. This signed pressure force provides outward balloon forces when the surface resides within the WM and inward forces when it resides outside the WM, thus forcing the surface to the GM/WM boundary. The advection force $\vec{F}_{\text {adv }}(\mathbf{x})$ in Eq. (6) is not needed. The weights for the two forces are chosen empirically to be $\omega_{R}=1$ and $\omega_{\kappa}=-0.02$.

The TGDM is initialized using the signed distance function computed from the topology-corrected 0.5 -isosurface of $\mu_{\mathrm{WM}}^{\prime}(\mathbf{x})$ and is run until convergence. The zero level set of the final level set function $\Phi_{\text {in }}$ gives the final estimate of the inner cortical surface. An explicit triangulation of this surface is computed by applying the CCMC isosurface algorithm (Han et al., 2003) to $\Phi_{\text {in }}$. TGDM guarantees that this surface has a spherical topology, which can be verified by checking the Euler number (Agoston, 1976) of the triangulated surface mesh. An example of a computed inner cortical surface is shown in Fig. 9a. The blue curves in Fig. 10 show the intersections of this estimated inner cortical surface with various (skull-stripped) MR image slices.

\section{Central cortical surface}

The central cortical surface is found by applying another TGDM, starting from $\Phi_{\text {in }}$ and with the zero level set of the evolving level set function $\Phi(\mathbf{x})$ being driven towards the central layer of the ACE-enhanced GM membership function $\mu_{\mathrm{GM}}^{\prime}$. To accomplish this, we follow the basic idea of (Xu et al., 1999) and use $\mu_{\mathrm{GM}}^{\prime}$ as an edge map in the computation of a GVF force, which then guides the deformable surface to the central surface of the GM. In particular, the GVF external force field $\vec{v}(\mathbf{x})$ is computed as the equilibrium solution of the following (vector) partial differential equation

$$
\begin{aligned}
\overrightarrow{v_{t}}(\mathbf{x}, t)= & c \nabla^{2} \vec{v}(\mathbf{x}, t) \\
& -\left(\vec{v}(\mathbf{x}, t)-\nabla \mu_{\mathrm{GM}}^{\prime}(\mathbf{x})\right)\left\|\nabla \mu_{\mathrm{GM}}^{\prime}(\mathbf{x})\right\|^{2},
\end{aligned}
$$

where $\overrightarrow{v_{t}}$ denotes the partial derivative of $\vec{v}(\mathbf{x}, t)$ with respect to $t$, $\nabla^{2}$ is the Laplacian operator, and $c$ is a weight, which we set to 0.2 . 


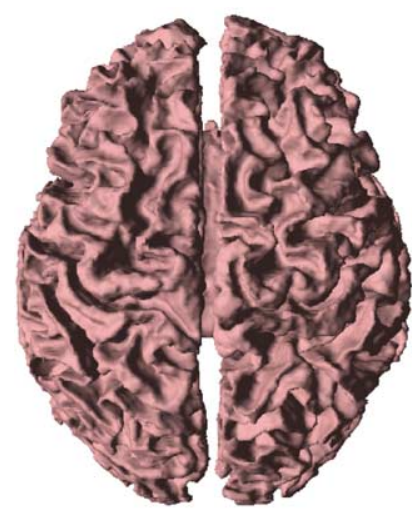

(a)

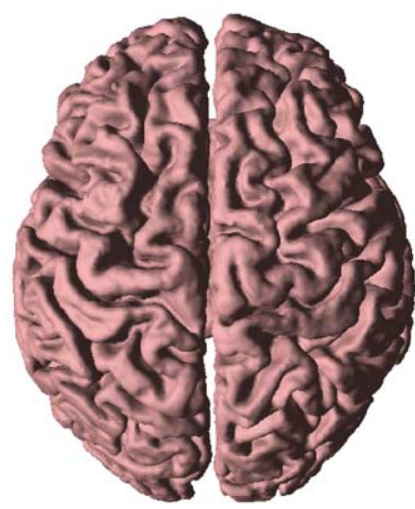

(b)

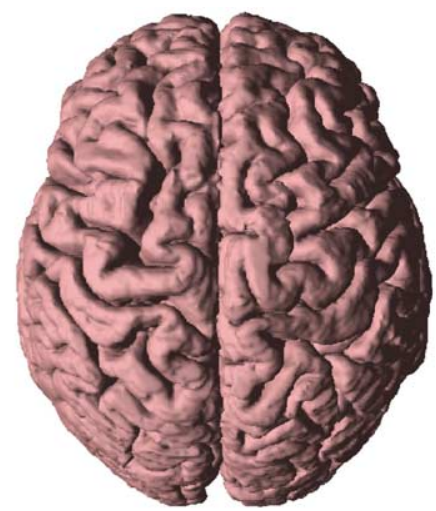

(c)

Fig. 9. Top view of the reconstructed (a) inner, (b) central, and (c) outer cortical surfaces derived from the same brain scan.

To make sure that the surface does not move out of the cortex, we also apply a regional pressure force similar to the one used in $\mathrm{Xu}$ et al. (1999),

$R_{\text {cent }}(\mathbf{x})= \begin{cases}0, & \text { if }\left\|2 \mu_{\mathrm{WM}}^{\prime}(\mathbf{x})+\mu_{\mathrm{GM}}^{\prime}(\mathbf{x})-1\right\|<0.5 \\ 2 \mu_{\mathrm{WM}}^{\prime}(\mathbf{x})+\mu_{\mathrm{GM}}^{\prime}(\mathbf{x})-1, & \text { otherwise. }\end{cases}$

This force pushes the surface outward if it is in the WM, inward if in the CSF, but has no effect within the GM. Thus, the surface finally moves toward the central surface of the GM under the influence of the GVF force alone. We also apply a small curvature force to keep the surface smooth. The relative strengths of the individual forces are determined by their coefficients, which we fix to be $\omega_{R}=\omega_{\dot{v}}=1$ and $\omega_{\kappa}=-0.02$ for all brain studies.

Using these internal and external forces, a slightly modified TGDM is initialized using $\Phi_{\text {in }}$ and run until convergence, yielding $\Phi_{\text {cent }}$. The small modification that is made is to apply an additional constraint guaranteeing that the resulting surfaces are properly nested - that is, the surface implied by $\Phi_{\text {cent }}$ is not inside that implied by $\Phi_{\text {in }}$. It turns out that this constraint is easy to apply: when updating the evolving level set function $\Phi(\mathbf{x})$ at any voxel $\mathbf{x}$, we prevent the value $\Phi(\mathbf{x})$ from becoming larger than $\Phi_{\text {in }}(\mathbf{x})$. By level set function convention (negative is inside), this constraint guarantees that the estimated central cortical surface is not inside the inner cortical surface at any point. An example of a computed central cortical surface is shown in Fig. 9b. The red curves in Fig. 10 show the intersections of this estimated central cortical surface with various (skull-stripped) MR image slices.

\section{Outer cortical surface}

The final TGDM model is designed with forces to find the outer cortical surface starting from the central cortical surface. The outer cortical surface should approximate the outer 0.5isosurface of the ACE-modified cortical GM membership function $\mu_{\mathrm{GM}}^{\prime}$. To remove ambiguity with the inner 0.5 -isosurface, we note that the desired surface is also given by the 0.5 isosurface of $\mu_{\mathrm{WM}}^{\prime}+\mu_{\mathrm{GM}}^{\prime}$, where the AutoFill WM membership is used to prevent possible confusion with the ventricles and subcortical GM. The image force is analogous to that used for the inner surface. In particular, we use a signed pressure force defined by

$R_{\text {out }}(\mathbf{x})=2\left(\mu_{\mathrm{GM}}^{\prime}(\mathbf{x})+\mu_{\mathrm{WM}}^{\prime}(\mathbf{x})\right)-1$, which makes the surface expand if it is inside the GM or WM and contract if it is in the CSF. We do not use an advection force but apply a small curvature force to keep the surface smooth. The weights are chosen empirically to be $\omega_{R}=1$ and $\omega_{\kappa}=-0.02$.

This TGDM algorithm is initialized using the central surface output $\Phi_{\text {cent }}$ and evolves $\Phi(\mathbf{x})$ until convergence, yielding $\Phi_{\text {out }}(\mathbf{x})$. To guarantee proper surface nesting, we impose an analogous constraint as that used for the central surface. In particular, when computing the evolving level set function $\Phi(\mathbf{x})$ using Eq. (8), we check and prevent its value from becoming larger than $\Phi_{\text {cent }}(\mathbf{x})$. CCMC is again used to extract an explicit triangulated outer cortical surface mesh from the final $\Phi_{\text {out }}$ after convergence. An example of a computed outer cortical surface is shown in Fig. 9c. The yellow curves in Fig. 10 show the intersections of this estimated outer cortical surface with various (skull-stripped) MR image slices.

\section{Results}

We have applied CRUISE with the same set of default parameters (as given in Methods when each component algorithm was presented) to nearly three hundred MR brain images obtained from the Baltimore Longitudinal Study on Aging (Resnick et al., 2000). Visual inspection of the surface reconstruction results has verified that the method worked successfully each time. In this section, we present some quantitative evaluations of the performance of CRUISE based on several carefully designed validation studies. We will also demonstrate the use of the surface reconstruction in studying the cortical geometry, which illustrates the potential of applying CRUISE in various neuroscience applications.

\section{Central surface landmarks}

In previous work, we developed a method to find the central surface of the cortex from MR images (Xu et al., 1999). The accuracy of our previous approach was evaluated (in part) using a set of 60 central surface landmark points, five on each hemisphere of six brains, placed manually. The distance from each landmark to the estimated central cortical surface served as a measure of accuracy. In the present study, we used five of these same six MR data sets and with most of the same landmarks. One subject (Subject 5 in Xu et al., 1999) was omitted because it is coronally 


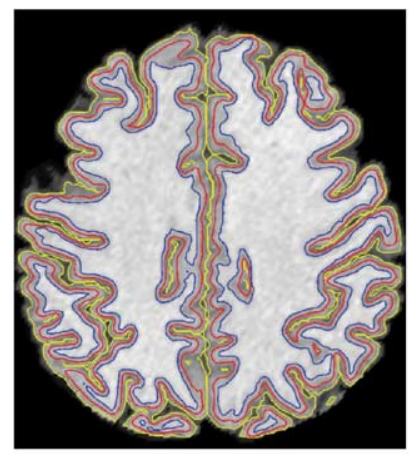

(a)

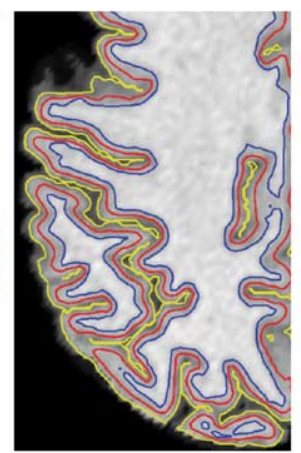

(b)

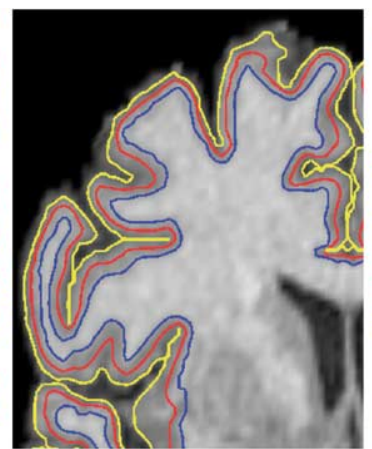

(c)

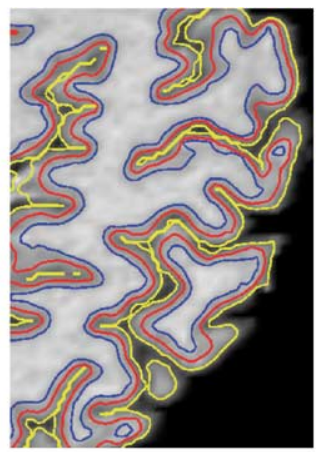

(d)

Fig. 10. (a) Three reconstructed surfaces displayed on an axial slice of the original MR data. (b) A zoom of the bottom-left corner of (a). (c, d) Zoomed views on coronal and sagittal slices, respectively. Blue: inner: red: central; yellow: outer.

acquired data with substantially different resolution and contrast characteristics. Fifteen landmark points were reselected because of anomalies discovered in the original picking process.

The landmark errors computed at 10 central surface landmark points for each of the five subjects are reported in Table 1. The errors are typically less than a voxel with a total average error of $0.51 \mathrm{~mm}$. For comparison, we recomputed the landmark errors of our previous method and have included the mean and standard deviations (SD) in the last two columns of Table 1. In comparison to our old method, CRUISE exhibits both improved overall accuracy and substantially increased stability, as indicated by the lower standard deviation value.

\section{Inner and outer surface landmarks}

Specifically for CRUISE, we conducted a comprehensive landmark-based study of accuracy for the inner and outer cortical surfaces. A graphical user interface was written to display selected slices of the raw MR brain volume. Within each view, a user ${ }^{2}$ was asked to pick 10 landmark points located on the GM/WM interface and on either a sulcal fundus, sulcal bank, or gyral crown. A region-of-interest box was used to indicate the selection region and within which the landmarks were picked. The user picked a total of 420 landmark points, corresponding to 14 fundi, 14 banks, and 14 gyral crowns near major sulci in a single brain across both hemispheres. An analogous procedure was used to pick 420 landmark points on the GM/CSF interface. Two brains were analyzed this way, neither being among the five used in the central surface study above.

CRUISE was used to find inner, central, and outer surfaces on the two subject brains in this study. The closest point from each landmark to the corresponding CRUISE surface was found and the distance between them computed (we use both signed and unsigned distances; when signed distance is used, negative values indicate the landmark point being inside the surface). We refer to these distances as landmark errors, although they are really just distances to a reference surface which happens to be our estimated surface. The landmark errors for the inner and outer surfaces of the two brains are shown in Table 2.

\footnotetext{
${ }^{2}$ This was the same user who picked the central surface landmark points in the previous section.
}

From Table 2, we see that, for the inner surface, the mean signed landmark error is between -0.34 and $0.36 \mathrm{~mm}$, and the overall mean absolute landmark error is about $0.46 \mathrm{~mm}$. These numbers are consistent with a claim that the inner surface has about $0.5-\mathrm{mm}$ accuracy and is not biased in any direction. Although the maximal landmark error is above $2 \mathrm{~mm}$, very few landmark points have errors this large. In fact, only between $10 \%$ and $12 \%$ of the landmark points are farther than $1 \mathrm{~mm}$ from the estimated inner surfaces, and less than $1 \%$ of them are farther than $2 \mathrm{~mm}$, which indicates that gross errors are not common. The numbers are very similar for the outer surface with the exception that the mean signed landmark error tends to be larger, in the range -0.68 to $0.48 \mathrm{~mm}$. The mean absolute landmark error is about $0.5 \mathrm{~mm}$. These numbers are consistent with a claim of perhaps $0.5-\mathrm{mm}$ accuracy and no bias in either direction. The number of landmark points farther than $1 \mathrm{~mm}$ from the estimated outer surfaces is still less than $12 \%$ of the total number, and the number of landmark points farther than $2 \mathrm{~mm}$ is less than $0.3 \%$. We note that there is a consistent negative mean signed landmark error at the sulcal fundi for both the inner and outer surfaces. This could suggest that both surfaces are being reconstructed inside the true surface in the fundus regions.

A more extensive study is underway to gain additional insights with statistical significance.

\section{Repeatability study}

In addition to being accurate, we would also like the cortical surfaces to be consistently reproducible in successive scans of the same subject. In fact, for longitudinal analysis, repeatability is a critical feature of a cortical reconstruction algorithm, since change detection is the main objective. To quantify the repeatability of CRUISE, three subjects were each scanned twice within a 30 -min period, the second time after repositioning in the scanner. CRUISE was applied to each of these MR data sets to generate the six sets of nested surfaces. The repeat scan surfaces were then aligned to the original surfaces using a modified Iterated Closest Point (ICP) algorithm (Besl and Mckay, 1992). In particular, our ICP algorithm used all three surfaces and sought to minimize the sum of distances from all points (on six triangulated meshes) to their respective correspondence. Also, the artificial surface points produced by AutoFill on the inferior aspect were ignored in the modified ICP process. The reason that we apply ICP to directly align the surfaces instead of using a volume registration method is because here we 
Table 1

Central surface landmark errors $(\mathrm{mm})$

\begin{tabular}{|c|c|c|c|c|c|c|c|c|c|}
\hline \multirow[t]{2}{*}{ Landmark } & \multicolumn{5}{|c|}{ Subject } & \multicolumn{2}{|c|}{ CRUISE } & \multicolumn{2}{|c|}{ Old method } \\
\hline & 1 & 2 & 3 & 4 & $5^{\mathrm{a}}$ & Mean & Std & Mean & Std \\
\hline $\mathrm{CS}_{1}$ & 0.52 & 0.47 & 0.81 & 0.37 & 0.40 & 0.51 & 0.18 & 0.69 & 0.74 \\
\hline $\mathrm{CS}_{2}$ & 0.08 & 0.40 & 0.49 & 0.06 & 0.28 & 0.26 & 0.19 & 0.98 & 0.77 \\
\hline $\mathrm{PCG}_{1}$ & 0.68 & 0.26 & 0.18 & 0.70 & 0.74 & 0.51 & 0.27 & 0.50 & 0.18 \\
\hline $\mathrm{PCG}_{2}$ & 0.63 & 0.11 & 0.48 & 0.36 & 0.65 & 0.45 & 0.22 & 0.63 & 0.48 \\
\hline $\mathrm{TLG}_{1}$ & 0.20 & 0.41 & 0.75 & 0.89 & 0.08 & 0.47 & 0.35 & 0.98 & 1.28 \\
\hline $\mathrm{TLG}_{2}$ & 1.27 & 2.35 & 0.54 & 0.75 & 0.44 & 1.07 & 0.78 & 1.92 & 1.25 \\
\hline $\mathrm{CALC}_{1}$ & 0.11 & 0.48 & 0.18 & 0.08 & 0.09 & 0.19 & 0.17 & 0.47 & 0.55 \\
\hline $\mathrm{CALC}_{2}$ & 0.82 & 0.13 & 0.70 & 0.28 & 0.49 & 0.48 & 0.29 & 1.68 & 2.34 \\
\hline $\mathrm{MFG}_{1}$ & 0.16 & 0.74 & 0.09 & 0.22 & 0.82 & 0.41 & 0.35 & 0.37 & 0.43 \\
\hline $\mathrm{MFG}_{2}$ & 0.30 & 1.61 & 0.46 & 0.50 & 0.78 & 0.73 & 0.52 & 0.65 & 0.56 \\
\hline Mean & 0.48 & 0.70 & 0.47 & 0.42 & 0.48 & 0.51 & - & 0.89 & - \\
\hline SD & 0.38 & 0.72 & 0.25 & 0.28 & 0.27 & - & 0.41 & - & 0.92 \\
\hline
\end{tabular}

CS, indicates fundus of central sulcus; PCG, crown of precentral gyrus; TLG, anterior point on temporal lobe gyrus; CALC, fundus of calcarine sulcus; MFG, medial frontal gyrus.

${ }^{a}$ Subject 5 was Subject 6 in Xu et al. (1999).

are interested in comparing the shapes of the estimated cortical surfaces. Directly aligning the surfaces avoids the possibility that the reported surface differences are biased by volume registration errors.

In Table 3, we show statistics derived from distances computed from the surface of the second scan to that of the first (after ICP registration). Overall, the mean signed distances for any surface are just a few hundredths of a millimeter. This indicates that the corresponding surfaces are well aligned, and there are no gross geometric errors between the two scans. The overall mean absolute distances are in the range $0.23-0.32 \mathrm{~mm}$, which is slightly less than the landmark errors reported in the previous section. There are smaller means and standard deviations on central cortical surface compared with those for inner and outer surfaces. This adds weight to the claim that the central surface can be found in a more robust fashion than either the inner or outer surface (Xu et al., 1999).

Although the average errors and standard deviations are reasonably small, there are regions where the aligned surfaces are separated by large distances. Table 3 indicates the percentage of analyzed surface (ignoring the inferior "AutoFill points") where the absolute distance is larger than 1 and $2 \mathrm{~mm}$. This is between $1 \%$ and $4 \%$ of the surface for $1 \mathrm{~mm}$ and less than $0.4 \%$ of the surface for $2 \mathrm{~mm}$. These distances are small, but of concern in longitudinal analysis where the changes sought might be in the neighborhood of these small percentages. We analyzed many of these regions manually and found that large errors can occur because of differences in skull stripping, AutoFill, topology correction, and
ACE. Skull stripping is particularly problematic because user interaction can yield erroneously removed GM or remaining dura, which can affect the positioning of all three surfaces, but particularly the central and outer surfaces. The remaining sources of error are often caused by a noise spike present in one data set but not the other and subtle differences in partial volume averaging due to the slight positioning differences between the two scans.

\section{Qualitative demonstrations}

We have demonstrated accurate and robust reconstruction of three cortical surfaces. The central surface, in particular, demonstrates accuracy and repeatability in the range of 0.5 and $0.25 \mathrm{~mm}$, respectively. We believe that the central surface is useful for characterization and analysis of the 2-D geometry of the cortex - for example, computation of curvatures (Cachia et al., 2003a; Zeng et al., 1999), geodesic distances (Cachia et al., 2003b; Rettmann et al., 2002), segmenting sulci or gyri (Behnke et al., 2003; Cachia et al., 2003b; Rettmann et al., 2002), surface flattening (Carman et al., 1995; Drury et al., 1996), and spherical mapping (Angenent et al., 1999; Fischl et al., 1999a; Tosun and Prince, 2001).

For example, on the top row of Fig. 11, we show the mean curvature of a central cortical surface displayed on the original surface, its partial flattening, and its spherical map. Mean curvature is commonly used to distinguish sulcal fundi (negative mean curvature) from gyral crowns (positive mean curvature). As another example, the second row of Fig. 11 shows the geodesic

Table 2

Landmark errors for inner and outer cortical surfaces (mm)

\begin{tabular}{|c|c|c|c|c|c|c|c|c|c|c|}
\hline \multirow[t]{2}{*}{ Region } & \multicolumn{5}{|l|}{ Subject 1} & \multicolumn{5}{|l|}{ Subject 2} \\
\hline & Mean (SD) & $\mathrm{SD}(\mathrm{SD})$ & Mean (AD) & $\mathrm{SD}(\mathrm{AD})$ & $\operatorname{Max}(\mathrm{AD})$ & Mean (SD) & $\mathrm{SD}(\mathrm{SD})$ & Mean (AD) & $\mathrm{SD}(\mathrm{AD})$ & Max AD \\
\hline Inner fundus & -0.34 & 0.55 & 0.49 & 0.42 & 1.70 & -0.33 & 0.53 & 0.47 & 0.42 & 2.17 \\
\hline Inner bank & -0.04 & 0.45 & 0.31 & 0.32 & 1.85 & -0.06 & 0.54 & 0.40 & 0.36 & 1.89 \\
\hline Inner gyrus & 0.36 & 0.71 & 0.58 & 0.54 & 2.29 & 0.07 & 0.63 & 0.54 & 0.33 & 1.56 \\
\hline Overall inner & -0.01 & 0.64 & 0.46 & 0.45 & 2.29 & -0.11 & 0.59 & 0.46 & 0.38 & 2.17 \\
\hline Outer fundus & -0.68 & 0.45 & 0.69 & 0.44 & 1.90 & -0.16 & 0.58 & 0.45 & 0.39 & 2.00 \\
\hline Outer bank & -0.50 & 0.34 & 0.51 & 0.33 & 1.41 & 0.30 & 0.37 & 0.35 & 0.32 & 1.32 \\
\hline Outer gyrus & -0.34 & 0.40 & 0.42 & 0.32 & 1.50 & 0.48 & 0.51 & 0.55 & 0.43 & 1.70 \\
\hline Overall outer & -0.51 & 0.42 & 0.54 & 0.38 & 1.90 & 0.21 & 0.56 & 0.45 & 0.39 & 2.00 \\
\hline
\end{tabular}

$\mathrm{SD}$, indicates signed distance $(\mathrm{mm})$; $\mathrm{AD}$, absolute distance $(\mathrm{mm})$. 
Table 3

Repeatability: scan 2 to scan 1 distances (mm)

\begin{tabular}{|c|c|c|c|c|c|c|c|c|}
\hline \multirow[t]{2}{*}{$\mathrm{T}$} & \multirow[t]{2}{*}{$\mathrm{S}$} & \multirow[t]{2}{*}{ Surface } & \multicolumn{2}{|l|}{$\mathrm{AD}$} & \multicolumn{2}{|l|}{$\mathrm{SD}$} & \multirow[t]{2}{*}{$>1 \mathrm{~mm}$} & \multirow[t]{2}{*}{$>2 \mathrm{~mm}$} \\
\hline & & & Mean & Std & Mean & Std & & \\
\hline A1 & A2 & inner & 0.32 & 0.31 & 0.03 & 0.44 & $3.4 \%$ & $0.3 \%$ \\
\hline B1 & B2 & inner & 0.27 & 0.26 & 0.03 & 0.37 & $2.1 \%$ & $0.2 \%$ \\
\hline $\mathrm{C} 1$ & $\mathrm{C} 2$ & inner & 0.31 & 0.33 & 0.04 & 0.45 & $3.5 \%$ & $0.4 \%$ \\
\hline \multicolumn{3}{|c|}{ All inner points } & 0.30 & 0.30 & 0.04 & 0.42 & $3.0 \%$ & $0.3 \%$ \\
\hline A1 & A2 & central & 0.26 & 0.27 & -0.01 & 0.37 & $2.2 \%$ & $0.2 \%$ \\
\hline B1 & B2 & central & 0.23 & 0.24 & 0.01 & 0.33 & $1.8 \%$ & $0.2 \%$ \\
\hline $\mathrm{C} 1$ & $\mathrm{C} 2$ & central & 0.26 & 0.28 & 0.00 & 0.38 & $2.4 \%$ & $0.3 \%$ \\
\hline \multicolumn{3}{|c|}{ All central points } & 0.25 & 0.27 & 0.00 & 0.36 & $2.2 \%$ & $0.3 \%$ \\
\hline A1 & A2 & outer & 0.31 & 0.32 & -0.05 & 0.35 & $3.7 \%$ & $0.4 \%$ \\
\hline B1 & B2 & outer & 0.26 & 0.33 & -0.08 & 0.39 & $3.0 \%$ & $0.4 \%$ \\
\hline $\mathrm{C} 1$ & $\mathrm{C} 2$ & outer & 0.25 & 0.28 & -0.06 & 0.37 & $2.5 \%$ & $0.3 \%$ \\
\hline \multicolumn{3}{|c|}{ All outer points } & 0.27 & 0.31 & -0.06 & 0.38 & $3.0 \%$ & $0.4 \%$ \\
\hline
\end{tabular}

distance measured from the outer cortex (which has zero geodesic distance and is colored gray). The third row of Fig. 11 shows the result of a watershed-based sulcal segmentation algorithm, which utilizes the geodesic distance measure (Rettmann et al., 2002).

The bottom row of Fig. 11 shows a cortical thickness map displayed on the central surface. Cortical thickness is computed using all three surfaces as follows. Suppose $\mathbf{x}$ is a point on the triangulated central cortical surface. Since our level set functions are signed distance functions with negative values on the interior, the distance at $\mathbf{x}$ to the inner cortical surface is given by $\Phi_{\text {in }}(\mathbf{x})$ and the distance to the outer cortical surface by $-\Phi_{\text {out }}(\mathbf{x})$. Therefore, we compute the cortical thickness at $\mathbf{x}$ using

Thickness $(\mathbf{x})=\Phi_{\text {in }}(\mathbf{x})-\Phi_{\text {out }}(\mathbf{x})$.

Since $\mathbf{x}$ will not lie on a $3-\mathrm{D}$ grid point in general, $\Phi_{\text {in }}(\mathbf{x})$ and $\Phi_{\text {out }}(\mathbf{x})$ are computed using trilinear interpolation. The computed thicknesses in general fall in the range of 1-5 mm, which agrees well with the literature (Beatty, 2001; Griffin, 1994; von Economo, 1929; Zilles, 1990).

\section{Computation times}

Given the preprocessed MR image and the $\mathrm{AC}$ and $\mathrm{PC}$ landmark points, the entire CRUISE process currently takes less than 25 min on a desktop computer with a Linux operating system (2.2 GHz, Intel Pentium4 processor) to reconstruct all the three cortical surfaces. FANTASM requires $4 \mathrm{~min} 30 \mathrm{~s}$. AutoFill takes less than $1 \mathrm{~min}$. The topology correction typically takes less than $2 \mathrm{~min}$, and ACE requires less than $2 \mathrm{~min}$. The TGDM for the inner surface takes less than $1 \mathrm{~min}$ due to the close initialization. The TGDM for the central surface takes about $10 \mathrm{~min}, 6 \mathrm{~min}$ of which is spent for the computation of the gradient vector flow (GVF) force. $^{3}$ The TGDM for the outer surface takes less than $4 \mathrm{~min}$.

\section{Discussion}

Many approaches have been proposed in the literature for the reconstruction of the cortex from MR brain images (Dale et al., 1999; Davatzikos and Bryan, 1996; Joshi et al., 1999; Kriegeskorte

\footnotetext{
3 A multigrid version of GVF was recently implemented, reducing the time by 5 min with no change in results.
}

and Goebel, 2001; MacDonald et al., 2000; Mangin et al., 1995; Sandor and Leahy, 1997; Shattuck and Leahy, 2002; Teo et al., 1997; Zeng et al., 1999; Xu et al., 1999). These approaches differ in their ability to capture the convoluted cortical geometry, reconstruction accuracy, robustness against imaging artifacts, computation time, topological correctness, and self-intersection avoidance. We now give a brief survey of several of these methods, and compare them to CRUISE.

Mangin et al. (1995) introduced a homotopically deformable region method to construct a topographical map of the cortex that preserved the correct topology. A major shortcoming of this method is that its accuracy is limited to the voxel level, and thus the method is not well suited for finding the pial surface. The same shortcoming is shared by another volumetric cortical reconstruction method proposed by Teo et al. (1997). In addition, the tissue segmentation methods in both approaches are far from optimal. Imaging artifacts such as intensity inhomogeneities and partial volume effects were not taken into consideration.

The work by Joshi et al., 1999 reconstructs the GM/WM surface by first segmenting brain tissues using a maximum likelihood classifier and then computing a surface representation of the GM/WM interface using an isosurface algorithm. The method handles the intensity inhomogeneity by performing the tissue segmentation locally within small neighborhoods of each voxel. This work was focused on the processing of macaque brain images, although the method was also tested on cryosectional image data from the Visible Human project. A major drawback of the method is that it requires a tedious manual editing step to correct the topology of the surface estimation, which can take up to several days, as indicated in the paper.

The work by Davatzikos and Bryan (1996) was among the first to apply 3-D deformable surface model to the cortical surface reconstruction problem. This work directly models the brain cortex as a convoluted sheet of finite width and applies a center-of-mass force to drive the surface to the geometric center of the cortical sheet. A major drawback of this method lies in its inability to fully capture cortical folds, and thus an extra step is needed to extract the detailed sulcal structures (Davatzikos and Bryan, 1996; Vaillant and Davatzikos, 1997). The results, however, no longer constitute a single coherent surface representation of the cortex.

The method developed by Dale et al. (1999) reconstructs both the inner and the outer cortical surfaces in a largely automatic manner. The method first corrects the intensity inhomogeneity 

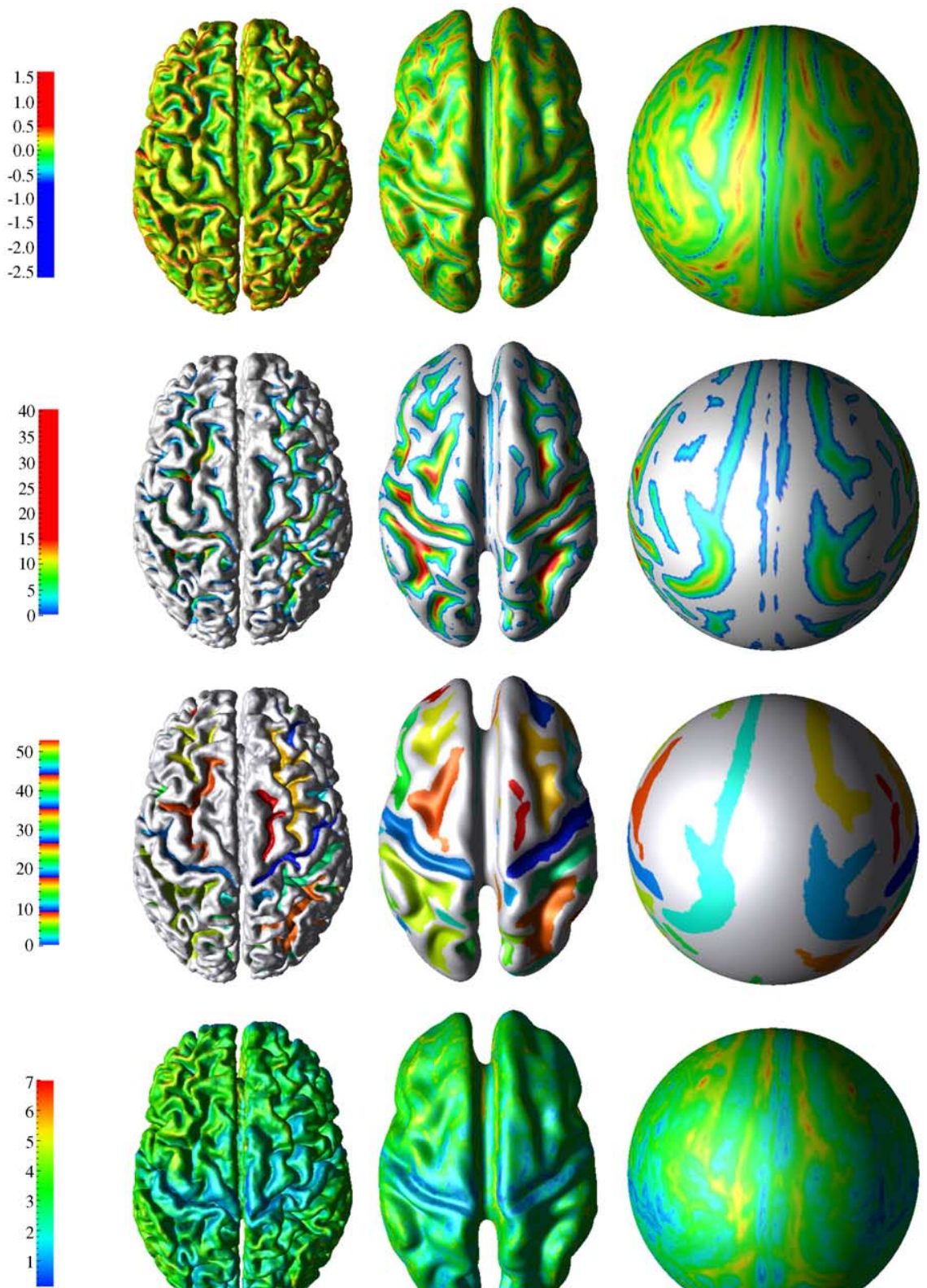

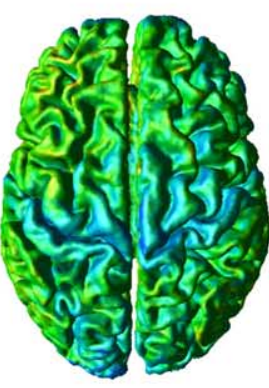

(b) central surface

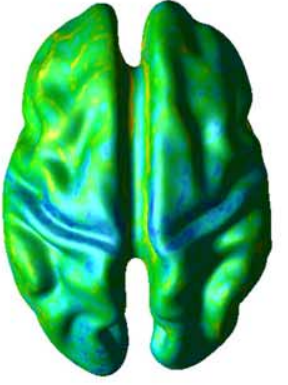

(c) partial flattening

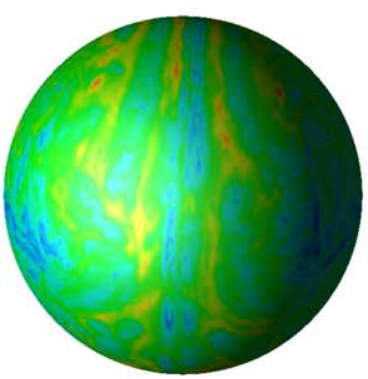

(d) spherical map

Fig. 11. (Top row) Mean curvature. (Second row) Geodesic distance from outer cortex. (Third row) Sulcal segmentation result. (Bottom) cortical thickness.

artifact by normalizing WM peaks across 2-D image slices. It then removes extracranial tissues using a deformable template model and labels the WM based on intensity and neighborhood information. Finally, a surface tessellation of the WM segmentation is computed and further deformed to find the GM/WM and GM/ CSF surfaces using a parametric deformable surface model. Although self-intersection is explicitly checked and prevented within the deformable surface model, the topology of the resulting surfaces is typically incorrect due to the lack of a topologically correct initial surface. As a result, the method requires a manual editing to remove topological defects from the deformable model outputs. We note that this manual editing was later replaced by an automatic topology correction method (Fischl et al., 2001), but this latter method is still very time-consuming. Another weakness of this method lies in the simple intensity thresholding-based tissue segmentation approach.

The approaches by Zeng et al. (1999) and by MacDonald et al. (2000) apply a coupled surface propagation strategy with a tight thickness constraint to get robust cortical surface reconstructions. In both methods, the inner and the outer surfaces are reconstructed simultaneously, and the thickness constraint keeps the two surfaces at close distance. A problem common to both methods is that the assumption of a constant cortical thickness can bias the estimation of the true thickness, as observed by some researchers (Kruggel and von Cramon, 2000; MacDonald et al., 2000). The method by Zeng et al. (1999) has an additional drawback that the topology of the final surfaces is typically incorrect, because the geometric deformable model method it applies lacks control of surface 
topology. The same drawback exits in another very similar approach proposed by Goldenberg et al. (2002). The method by MacDonald et al. (2000) guarantees the correct surface topology by using a parametric deformable surface model and initializing it with two ellipsoidal surfaces. But the method has to rely on a very time-consuming procedure to prevent surface self-intersections during the surface deformation, and the overall computation time typically reaches near $30 \mathrm{~h}$, as reported in the paper.

Kriegeskorte and Goebel (2001) proposed using a similar approach as that of Dale et al. (1999) to reconstruct the inner and the outer surfaces of the brain cortex but developed an automatic topology correction algorithm to replace the manual editing step. The paper focused mainly on the topology correction method, while the performance of the overall cortical surface reconstruction method was barely discussed.

Shattuck and Leahy (2002) developed a system called BrainSuite for extracting the GM/WM interface from MR brain images. It first extracts the brain using a method proposed by Sandor and Leahy (1997). After intensity inhomogeneity correction, the extracted brain volume is segmented into six classes that include both pure tissue classes and mixtures arising from the partial volume effect. An automatic topology correction is then performed on a binarized WM volume, which is followed by an isosurface algorithm to get the WM boundary surface with the correct topology. It should be noted that without the smoothness constraint as in a deformable model method, an isosurface type of approach is likely to create highly irregular surface estimates due to its sensitivity to image noise. Note also that an isosurface method is unsuitable for finding the central cortical surface, since the central surface cannot be defined as an isosurface of any tissue segmentation. The BrainSuite method, for example, does not provide estimates for the central and pial surfaces, and the GM/WM surface itself is insufficient to fully characterize the geometry of the cortex.

Another cortical surface reconstruction method was previously reported by our research group in (Xu et al., 1999), the overall design strategy of which is inherited by the current CRUISE method. The CRUISE method, however, addressed several important limitations in the previous approach. First, the tissue classification algorithm used by Xu et al. (1999), although robust to intensity inhomogeneity, can be sensitive to image noise. Second, the method of Xu et al. (1999) relies on a median filtering approach for WM topology correction, which tends to oversmooth the initial surface and is not guaranteed to converge. Third, the method of Xu et al. (1999) has difficulty in capturing narrow sulci due to the partial volume effect. Fourth, the parametric deformable model used in Xu et al. (1999), although preserves surface topology, may produce surface selfintersections. Finally, Xu et al. (1999) find only the central cortical surface. Overall, the new developments in CRUISE lead to much better accuracy (as demonstrated in Results), largely increased robustness to imaging artifacts, and largely reduced amount of manual interaction and computation time.

Examining the cortical thickness map in Fig. 11 reveals an apparent disagreement with histological studies: Fig. 11 shows a overall thinner central sulcus area than surrounding regions, while histological studies claim that the primary motor area (i.e., the anterior bank of the central sulcus) should be one of the thickest regions over the whole cerebral cortex. After some investigation, we found that this disagreement is not a particular failure of our individual method. Instead, we have found that the thickness maps produced by other cortical reconstruction methods also consistently show a thinner central sulcus area (whether a thickness constraint is imposed during the cortical reconstruction or not). For example, the same trend is clearly seen in Fig. 17 of MacDonald et al. (2000) and Fig. 1 of Lerch et al. (2002). It can also be seen in Fig. 12 of Zeng et al. (1999) and Fig. 1 of Salat et al. (2004). A plausible explanation for this problem is that the central sulcus region has significantly shorter T1 relaxation time than other cortical regions (cf. Steen et al., 2000), and it also has significant T2 shortening with respect to aging (cf. Korogi et al., 1997). The short T1 and T2 in this region lead to abnormal GM intensity and lack of GM/WM contrast in an acquired MR brain image and thus cause problem in correctly locating the GM/WM interface. To address this problem, one may need to design new MR imaging protocols to get better images or to incorporate a cortical T1 and T2 map into the cortical reconstruction procedure. On the other hand, the results produced by our CRUISE method have been shown to be highly reproducible and represent well the underlying data. Thus, it can still serve as a good starting point for analyzing the cortical geometry.

\section{Conclusion and future work}

In this paper, we have described a new approach for brain cortex segmentation that reconstructs all three key representative surfaces of the cortex. The method, called CRUISE, is computationally fast and produces surfaces that are geometrically accurate, have the correct topology, and do not self-intersect or mutually intersect. CRUISE provides a full cortex characterization in a nearly fully automatic fashion, which should make it possible to conduct sophisticated neuroanatomical studies that involve large amount of imaging data. A more extensive evaluation of the accuracy of the method for optimization of its parameters is under investigation and will be reported soon in a separate paper.

\section{Acknowledgments}

The authors would like to thank Dr. Susan Resnick for providing the BLSA data and for discussions on validation studies. We would also like to thank Ms. Daphne Yu and Mr. Xiaodong Tao for providing software that was used in the validation studies.

This work was supported in part by NIH/NINDS Grant R01NS37747.

\section{References}

Agoston, M.K., 1976. Algebraic Topology-A First Course. Marcel Dekker Inc., New York.

Angenent, S., Haker, S., Tannenbaum, A., Kikinis, R., 1999. On the Laplace-Beltrami operator and brain surface flattening. IEEE Trans. Med. Imag. 18, 700-711.

Beatty, J., 2001. The Human Brain: Essentials of Behavioral Neuroscience. Sage Publications Inc., California.

Behnke, K.J., Rettmann, M.E., Pham, D.L., Shen, D., Davatzikos, C., Resnick, S.M., Prince, J.L., 2003. Automatic classification of sulcal regions of the human brain cortex using pattern recognition. Proc. SPIE: Medical Imaging.

Bes1, P.J., Mckay, N.D., 1992. A method for registration of 3-D shapes. IEEE Trans. Pattern Anal. Mach. Intell. 14, 239-256.

Bezdek, J.C., Hall, L.O., Clarke, L.P., 1993. Review of MR image segmentation techniques using pattern recognition. Med. Phys. 20, $1033-1048$. 
Cachia, A., Mangin, J.F., Rivière, D., Kherif, F., Boddaert, N., Andrade, A., Papadopoulos-Orfanos, D., Poline, J.-B., Bloch, I., Zilbovicius, M., Sonigo, P., Brunelle, F., Régis, J., 2003a. A primal sketch of the cortex mean curvature: a morphogenesis based approach to study the variability of the folding patterns. IEEE Trans. Med. Imag. 22, 754-765.

Cachia, A., Mangin, J.F., Rivière, D., Papadopoulos-Orfanos, D., Kherif, F., Bloch, I., Régis, J., 2003b. A generic framework for the parcellation of the cortical surface into gyri using geodesic Voronöi; diagrams. Med. Image Anal. 7, 403-416.

Cachier, P., Mangin, J.F., Pennec, X., Rivière, D., Papadopoulos-Orfanos, D., Régis, J., Ayache, N., 2001. Multisubject non-rigid registration of brain MRI using intensity and geometric features. In: Niessen, W. Viergever, M. (Eds.), Proc. MICCAI 2001, LNCS 2208. Springer Verlag, Berlin Heidelberg, pp. 734-742.

Carman, G.J., Drury, H.A., van Essen, D.C., 1995. Computational methods for reconstructing and unfolding the cerebral cortex. Cereb. Cortex 5, $506-517$.

Collins, D.L., Zijdenbos, A.P., Kollokian, V., Sled, J.G., Kabani, N.J., et al., 1998. Design and construction of a realistic digital brain phantom. IEEE Trans. Med. Imag. 17, 463-468. (http://www.bic.mni.mcgill.ca/ brainweb)

Dale, A.M., Sereno, M.I., 1993. Improved localization of cortical activity by combining EEG and MEG with MRI cortical surface reconstruction: a linear approach. J. Cogn. Neurosci. 5 (2), 162-176.

Dale, A.M., Fischl, B., Sereno, M.I., 1999. Cortical surface-based analysis I: segmentation and surface reconstruction. NeuroImage 9, 179-194.

Davatzikos, C., 1997. Spatial transformation and registration of brain images using elastically deformable models. Comput. Vision Image Underst. 66, 207-222.

Davatzikos, C., Bryan, N., 1996. Using a deformable surface model to obtain a shape representation of the cortex. IEEE Trans. Med. Imag. 15 (6), 785-795.

Drury, H.A., Essen, D.C.V., Anderson, C.H., Lee, W.C., Coogan, T.A., Lewis, J.W., 1996. Computerized mapping of the cerebral cortex: a multiresolution flattening method and a surface-based coordinate system. J. Cogn. Neurosci. 8, 1-28.

Essen, D.C., Van Lewis, J.W., Drury, H.A., Hadjikhani, N., Tootell, R.B.H., Bakircioglu, M., Miller, M.I., 2001. Mapping visual cortex in monkeys and humans using surface-based atlases. Vision Res. 41, 1359-1378.

Fischl, B., Sereno, M.I., Dale, A.M., 1999a. Cortical surface-based analysis II: inflation, flattening, and a surface-based coordinate system. NeuroImage 9, 195-207.

Fischl, B., Sereno, M.I., Tootell, R.B.H., Dale, A.M., 1999b. Highresolution intersubject averaging and a coordinate system for the cortical surface. Hum. Brain Mapp. 8, 272-284.

Fischl, B., Liu, A., Dale, A.M., 2001. Automated manifold surgery: constructing geometrically accurate and topologically correct models of the human cerebral cortex. IEEE Trans. Med. Imag. 20 (1), 70-80.

Goldenberg, R., Kimmel, R., Rivlin, E., Rudzsky, M., 2002. Cortex segmentation: a fast variational geometric approach. IEEE Trans. Med. Imag. 21, 1544-1551.

Goldszal, A.F., Davatzikos, C., Pham, D.L., Yan, M.X.H., Bryan, R.N., Resnick, S.M., 1998. An image processing system for qualitative and quantitative volumetric analysis of brain images. J. Comput. Assist. Tomogr. 22 (5), $827-837$.

Griffin, L.D., 1994. The intrinsic geometry of the cerebral cortex. J. Theor. Biol. 166 (3), 261-273

Grimson, W.E.L., Ettinger, G., Kapur, T., Leventon, M., Wells, W., Kikinis, R., 1998. Utilizing segmented MRI data in image-guided surgery. Int. J. Pattern Recogn. Artif. Intell. 11, 1367-1397.

Han, X., Xu, C., Braga-Neto, U., Prince, J.L., 2001. Graph-based topology correction for brain cortex segmentation. Proceedings of the XVIIth International Conference on Information Processing in Medical Imaging (IPMI'01). Springer Verlag, Berlin, pp. 395-401.

Han, X., Xu, C., Rettmann, M.E., Prince, J.L., 2001b. Automatic segmentation editing for cortical surface reconstruction. Proc. of SPIE Medical Imaging, vol. 4322. SPIE Press, Bellingham, WA, pp. 194-203.
Han, X., Xu, C., Tosun, D., Prince, J.L., 2001c. Cortical surface reconstruction using a topology preserving geometric deformable model. Proceedings of the 5th IEEE Workshop on Mathematical Methods in Biomedical Image Analysis (MMBIA2001). IEEE Press, Kauai, Hawaii, pp. 213-220

Han, X., Xu, C., Braga-Neto, U., Prince, J.L., 2002. Topology correction in brain cortex segmentation using a multiscale, graph-based algorithm. IEEE Trans. Med. Imag. 21, 109-121.

Han, X., Xu, C., Prince, J.L., 2003. A topology preserving level set method for geometric deformable models. IEEE Trans. Pattern Anal. Mach. Intell. 25, 755-768.

Hellier, P., Barillot, C., 2003. Coupling dense and landmark-based approaches for nonrigid registration. IEEE Trans. Med. Imag. 22, $217-227$

Jaume, S., Macq, B., Warfield, S.K., 2002. Labeling the brain surface using a deformable multiresolution mesh. In: Dohi, T., Kikinis, R. (Eds.), Proc. MICCAI 2002, LNCS 2488. Springer Verlag, Berlin Heidelberg, pp. $451-458$

Joshi, M., Cui, J., Doolittle, K., Joshi, S., Essen, D., Wang, L., Miller, M.I., 1999. Brain segmentation and the generation of cortical surfaces. NeuroImage 9, 461-476.

Kapur, T., Grimson, W.E.L., Wells, W.M., Kikinis, R., 1996. Segmentation of brain tissue from magnetic resonance images. Med. Image Anal. 1 (2), 109-127.

Kim, J., Crespo-Facorro, B., Andreasen, N.C., O’Leary, D.S., Zhang, B., Harris, G., Magnotta, V.A., 2000. An MRI-based parcellation method for the temporal lobe. NeuroImage 11 (4), 271-288.

Korogi, Y., Hirai, T., Komohara, Y., et al., 1997. T2 shortening in the visual cortex: effect of aging and cerebrovascular disease. Am. J. Neuroradiol. $18,711-714$

Kriegeskorte, N., Goebel, R., 2001. An efficient algorithm for topologically correct segmentation of the cortical sheet in anatomical MR volumes. NeuroImage 14, 329-346.

Kruggel, F., von Cramon, D.Y., 1999. Alignment of magnetic-resonance brain datasets with the stereotactical coordinate system. Med. Image Anal. 3, 175-185.

Kruggel, F., von Cramon, D.Y., 2000. Measuring cortical thickness. Proc. 4th IEEE Workshop on Mathematical Methods in Biomedical Image Analysis (MMBIA2000). IEEE Press, Hilton Head Island, SC, pp. $154-161$

Kruggel, F., Brückner, M.K., Arendt, T., Wiggines, C.J., von Cramon, D.Y., 2003. Analyzing the neocortical fine-structure. Med. Image Anal. 7, $251-264$

Lemoine, D., Barillot, C., Gibaud, B., Pasqualini, E., 1991. An anatomicalbased 3D registration system of multimodality and atlas data in neurosurgery. Lecture Notes in Computer Science, vol. 511. SpringerVerlag, Berlin, pp. 154-164.

Lerch, J., Pruessner, J., Zijdenbos, A., Teipel, S.T., Buerger, K., Hampel, H., Evans, A.C., 2002. Changes in cortical integrity in Alzheimer's disease. Proc. 8th Int. Conf. Functional Mapping of the Human Brain, Sendai, Japan.

MacDonald, D., Kabani, N., Avis, D., Evans, A.C., 2000. Automated 3-D extraction of inner and outer surfaces of cerebral cortex from MRI. NeuroImage 12, 340-356.

Magnotta, V.A., Andreasen, N.C., Schultz, S.K., Harris, G., Cizadlo, T., Heckel, D., Nopoulos, P., Flaum, M., 1999. Quantitative in vivo measurement of gyrification in the human brain: changes associated with aging. Cereb. Cortex 9, 151-160.

Manceaux-Demiau, A., Bryan, R.N., Davatzikos, C., 1998. A probabilistic ribbon model for shape analysis of the cerebral sulci: application to the central sulcus. J. Comput. Assist. Tomogr. 22 (6), 962-971.

Mangin, J.-F., Frouin, V., Bloch, I., Regis, J., Lopez-Krahe, J., 1995. From 3D magnetic resonance images to structural representations of the cortex topography using topology preserving deformations. J. Math. Imaging Vision 5, 297-318.

Pham, D.L., 2001. Robust fuzzy segmentation of magnetic resonance images. Proceedings of the Fourteenth IEEE Symposium on Com- 
puter-Based Medical Systems (CBMS2001). IEEE Press, Somerset, NJ, pp. $127-131$

Pham, D.L., Prince, J.L., 1999. Adaptive fuzzy segmentation of magnetic resonance images. IEEE Trans. Med. Imag. 18 (9), 737-752.

Pham, D.L., Han, X., Rettmann, M.E., Xu, C., Tosun, D., Resnick, S.M., Prince, J.L., 2002. New approaches for measuring changes in the cortical surface using an automatic reconstruction algorithm. Proc. SPIE Med. Imag., vol. 4684. SPIE Press, San Diego, CA, pp. 191-200.

Resnick, S.M., Goldszal, A.F., Davatzikos, C., Golski, S., Kraut, M.A., Metter, E.J., Bryan, R.N., Zonderman, A.B., 2000. One-year age changes in MRI brain volumes in older adults. Cereb. Cortex 10 (5), $464-472$.

Rettmann, M.E., Han, X., Xu, C., Prince, J.L., 2002. Automated sulcal segmentation using watersheds on the cortical surface. NeuroImage 15 , $329-344$

Rivière, D., Mangin, J.F., Papadopoulos-Orfanos, D., Martinez, J., Frouin, V., Régis, J., 2002. Automatic recognition of cortical sulci of the human brain using a congregation of neural networks. Med. Image Anal. 6, $77-92$.

Salat, D.H., Buckner, R.L., Snyder, A.Z., Greve, D.N., Desikan, R.S.R., Busa, E., Morris, J.C., Dale, A.M., Fischl, B., 2004. Thinning of the cerebral cortex in aging. Cereb. Cortex 14, 721-730.

Sandor, S., Leahy, R., 1997. Surface-based labeling of cortical anatomy using a deformable atlas. IEEE Trans. Med. Imag. 16, 41-54.

Sethian, J.A., 1999. Level Set Methods and Fast Marching Methods, second ed. Cambridge Univ. Press, Cambridge, UK.

Shattuck, D.W., Leahy, R.M., 1999. Topological refinement of volumetric data. Proceedings of the SPIE, vol. 3661. SPIE Press, San Diego, USA, pp. $204-213$.

Shattuck, D.W., Leahy, L.R., 2001. Graph based analysis and correction of cortical volume topology. IEEE Trans. Med. Imag. 20, 1167-1177.

Shattuck, D.W., Leahy, R.M., 2002. BrainSuite: an automated cortical surface identification tool. Med. Image Anal. 6, 129-142.

Steen, R.G., Reddick, W.E., Ogg, R.J., 2000. More than meets the eye: significant regional heterogeneity in human cortical T1. Magn. Reson. Imaging 18, 361-368.

Talairach, J., Tournoux, P., 1988. Co-Planar Stereotaxic Atlas of the Human Brain. Thieme Medical Publisher Inc., Stuttgart, New York.

Tao, X., Prince, J.L., Davatzikos, C., 2002. Using a statistical shape model to extract sulcal curves on the outer cortex of the human brain. IEEE Trans. Med. Imag. 21, 513-524.
Teo, P.C., Sapiro, G., Wandell, B.A., 1997. Creating connected representations of cortical gray matter for functional MRI visualization. IEEE Trans. Med. Imag. 16 (6), 852-863.

Thompson, P.M., MacDonald, D., Mega, M.S., Holmes, C.J., Evans, A.C., Toga, A.W., 1997. Detection and mapping of abnormal brain structure with a probabilistic atlas of cortical surfaces. J. Comput. Assist. Tomogr. 21, 567-581.

Thompson, P.M., Mega, M.S., Woods, R.P., Zoumalan, C.I., Lindshield, C.J., Blanton, R.E., Moussai, J., Holmes, C.J., Cummings, J.L., Toga, A.W., 2001. Cortical changes in Alzheimer's disease detected with a diseasespecific population-based brain atlas. Cereb. Cortex 11 (1), 1-16.

Tosun, D., Prince, J.L., 2001. Hemispherical map for the human brain cortex. Proc. of SPIE Medical Imaging, vol. 4322. SPIE Press, Bellingham, WA, pp. 290-300.

Vaillant, M., Davatzikos, C., 1997. Finding parametric representations of the cortical sulci using an active contour model. Med. Image Anal. 1 (4), 295-315.

von Economo, C., 1929. The Cytoarchitectonics of the Human Cerebral Cortex. Oxford Univ. Press, London.

Xu, C., Prince, J.L., 1998. Snakes, shapes, and gradient vector flow. IEEE Trans. Image Process. 7 (3), 359-369.

Xu, C., Pham, D.L., Rettmann, M.E., Yu, D.N., Prince, J.L., 1999. Reconstruction of the human cerebral cortex from magnetic resonance images. IEEE Trans. Med. Imag. 18 (6), 467-480.

Xu, C., Han, X., Prince, J.L., 2000. Improving cortical surface reconstruction accuracy using an anatomically consistent gray matter representation. Proc. NeuroImage Hum. Brain Map., vol. 11. Academic Press, San Diego, CA, p. S581.

Xu, C., Yezzi, A., Prince, J.L., 2001. A summary of geometric level-set analogues for a general class of parametric active contour and surface models. Proc. of 1st IEEE Workshop on Variational and Level Set Methods in Computer Vision. IEEE Press, Somerset, NJ, pp. 104-111.

Yezzi, A.J., Prince, J.L., 2003. An evolution PDE approach for computing tissue thickness. IEEE Trans. Med. Imag. 22 (10), 1332-1339.

Zeng, X., Staib, L.H., Schultz, R.T., Duncan, J.S., 1999. Segmentation and measurement of the cortex from 3D MR images using coupled surfaces propagation. IEEE Trans. Med. Imag. 18, 100-111.

Zhang, J., 1992. The mean field theory in EM procedures for Markov random fields. IEEE Trans. Signal Process. 40 (10), 2570-2583.

Zilles, K., 1990. Cortex in the Human Nervous System. Academic Press, San Diego, CA. 